\title{
High-Fidelity Modeling of Multirotor Aerodynamic Interactions for Aircraft Design
}

\author{
Eduardo Alvarez \\ Brigham Young University, edo.alvarezr@gmail.com \\ Andrew Ning \\ Brigham Young University, aning@byu.edu
}

Follow this and additional works at: https://scholarsarchive.byu.edu/facpub

Part of the Mechanical Engineering Commons

\section{Original Publication Citation}

Alvarez, E. J., and Ning, A., "High-Fidelity Modeling of Multirotor Aerodynamic Interactions for Aircraft Design," AIAA Journal, Aug. 2020. doi:10.2514/1.J059178

\section{BYU ScholarsArchive Citation}

Alvarez, Eduardo and Ning, Andrew, "High-Fidelity Modeling of Multirotor Aerodynamic Interactions for Aircraft Design" (2020). Faculty Publications. 4179.

https://scholarsarchive.byu.edu/facpub/4179

This Peer-Reviewed Article is brought to you for free and open access by BYU ScholarsArchive. It has been accepted for inclusion in Faculty Publications by an authorized administrator of BYU ScholarsArchive. For more information, please contact ellen_amatangelo@byu.edu. 


\title{
High-fidelity Modeling of Multirotor Aerodynamic Interactions for Aircraft Design
}

\author{
Eduardo J. Alvarez * and Andrew Ning. ${ }^{\dagger}$ \\ Brigham Young University, Provo, Utah, 84602
}

Electric aircraft technology has enabled the use of multiple rotors in novel concepts for urban air mobility. However, multirotor configurations introduce strong aerodynamic and aeroacoustic interactions that are not captured through conventional aircraft design tools. In this paper we explore the capability of the viscous vortex particle method (VPM) to model multirotor aerodynamic interactions at a computational cost suitable for conceptual design. A VPM-based rotor model is introduced along with recommendations for numerical stability and computational efficiency. Validation of the individual rotor is presented in both hovering and forward-flight configurations at low, moderate, and high Reynolds numbers. Hovering multirotor predictions are compared to experimental measurements, evidencing the suitability of the proposed model to capture the thrust drop and unsteady loading produced by rotor-onrotor interactions.

\section{Introduction}

Technology convergence in the past ten years has opened a new design space in electric aircraft, enabling the use of distributed propulsion and electrical vertical takeoff and landing (eVTOL) for urban air mobility [1-4]. The concept of eVTOL in an urban setting is forecasted to evolve into a $\$ 1.5$ trillion "flying car" industry by the year 2040 [5]. However, this unconventional aircraft configuration poses technical challenges that still remain to be solved [6]. A strong noise signature $[7,8]$ and a complicated transition maneuver $[9,10]$ are examples of the challenges encountered in eVTOL aircraft, both stemming in some degree from the aerodynamic interactions between rotors and lifting surfaces [11]. Furthermore, these interactions are common across quadrotor, tilt-rotor, tilt-wing, and distributed propulsion concepts making use of multiple rotors. As an example of eVTOL configuration, Fig. 2 shows NASA's GL-10 tilt-wing prototype aircraft during vertical takeoff, prior to transitioning into wing-borne forward flight.

The use of multiple rotors operating in close proximity introduces strong aerodynamic interactions that are not well understood, are not captured through conventional design tools, and need to be addressed in the conceptual design stage [12-15]. Experimental studies of side-by-side rotors in hover show a drop in thrust of 3-4\% [16-19] due to rotor-on-rotor interactions. More remarkably, Zhou et al. [20] and Ning [21] measured a noise increase of $3 \mathrm{~dB}$ associated with unsteady loading as the distance between rotors is decreased. In edgewise forward flight, Stokkermans et al. [22] found that the interactions can become as drastic as to drop the thrust by $30 \%$. Wake mixing and the unsteady

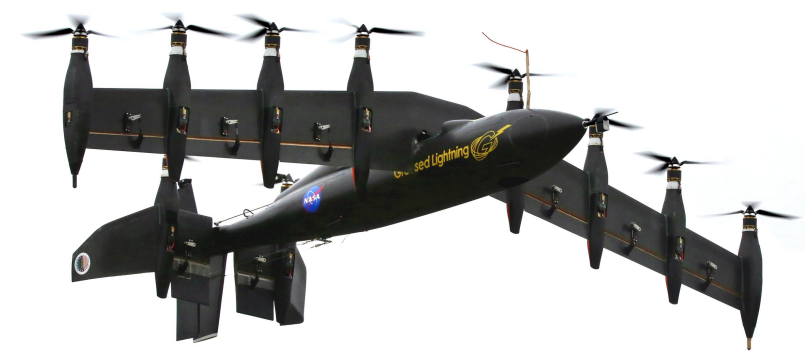

Fig. 2 NASA GL-10 eVTOL prototype aircraft during vertical takeoff. Credits: NASA Langley/David C. Bowman.

\footnotetext{
*Doctoral Candidate, Department of Mechanical Engineering, ealvarez@byu.edu, AIAA Member.

${ }^{\dagger}$ Assistant Professor, Department of Mechanical Engineering, aning@byu.edu, AIAA Senior Member.
} 
dynamics responsible for these effects are not captured through low-fidelity models used in conventional design tools. Multiple panel and filament methods have been developed for the modeling of unsteady free wakes, but experience shows that the requirement of preserving wake connectivity makes them ill-conditioned for the modeling of wake mixing [23]. These interactions can be analyzed through conventional computational fluid dynamics (CFD) approaches, like Reynolds-averaged Navier-Stokes (RANS) or large-eddy simulations (LES) [24-29], but computational cost makes them prohibitive for design space exploration. Hence, the ability to rapidly assess the performance of these design concepts, with sufficient fidelity, is a current weakness of the nascent eVTOL industry.

In this study we investigate the accuracy of the viscous vortex particle method [31, 32] (VPM) in modeling rotor-on-rotor aerodynamic interactions in a side-by-side configuration as encountered in tilt-wing, quadrotor, and distributed propulsion aircraft. The viscous VPM is a mesh-free CFD method for the numerical solution of the Navier-Stokes equations in their vorticity form. The VPM is a direct numerical simulation that efficiently preserves vortical structures in a Lagrangian scheme, eliminates the complexities of mesh generation, is absent of the numerical dissipation associated with mesh-based CFD, and is one to three orders of magnitude faster than RANS or LES*. This method has been shown to achieve a continuum of fidelity levels across resolved scales, from medium fidelity apt for conceptual-design [33-40] to high fidelity apt for fundamental fluid dynamics research [41-43]. Fig. 3 shows a mid-fidelity simulation of a full-aircraft configuration and a high-fidelity simulation of wake mixing in side-by-side propellers from previous work by the authors [30]. Furthermore, the method has been shown to be CPU and GPU parallelizable, and scalable in heterogeneous systems for high performance computing [44-46]. In previous work by the authors, the computational speed of the VPM has been demonstrated in a parametric study comprised of 1000+ multirotor simulations, requiring a wall-clock time of less than four days on a desktop computer with an average of four minutes per simulation [47]. Thus, coupled with an acoustics solver, the VPM approach could enable high-fidelity aerodynamic and aeroacoustic predictions capturing multirotor interactions during conceptual design.

The viscous VPM has been used in the rotorcraft community for the past two decades [48-52] demonstrating its efficiency in modeling wake-dominated problems, especially in the cases of coaxial rotors [53-55] and edgewise forward flight $[42,50,56-60]$. More recently, Tan et al. modeled the interactions between tandem rotors subjected to ground effect [61] and simulated the interactions between a tilt-rotor aircraft and a tandem-rotor helicopter while simultaneously landing on a ship deck [62], demonstrating the capacity of the VPM to capture intricate aerodynamic interactions. When coupled with the acoustic Ffowcs Williams-Hawkings equation, VPM-based simulations of isolated rotors have shown good agreement between experimental and predicted noise radiated from unsteady airloads, high-speed impulsive effects, and blade-vortex interactions [49, 63, 64].

Even though VPM has been extensively used in coaxial rotors and rotorcraft forward flight, little work has been done in modeling the side-by-side multirotor configuration encountered in eVTOL. This case is especially challenging because it needs to capture rotor-on-rotor interactions across the entire flight envelope of the eVTOL aircraft, from hover during takeoff to low and high advance ratios during transition and cruise, at both low and high Reynolds numbers [10]. In addition to performance predictions, it is also crucial to capture the unsteady loading introduced by rotor-on-rotor interactions in order to lay the groundwork for future noise predictions [65]. In this study we introduce
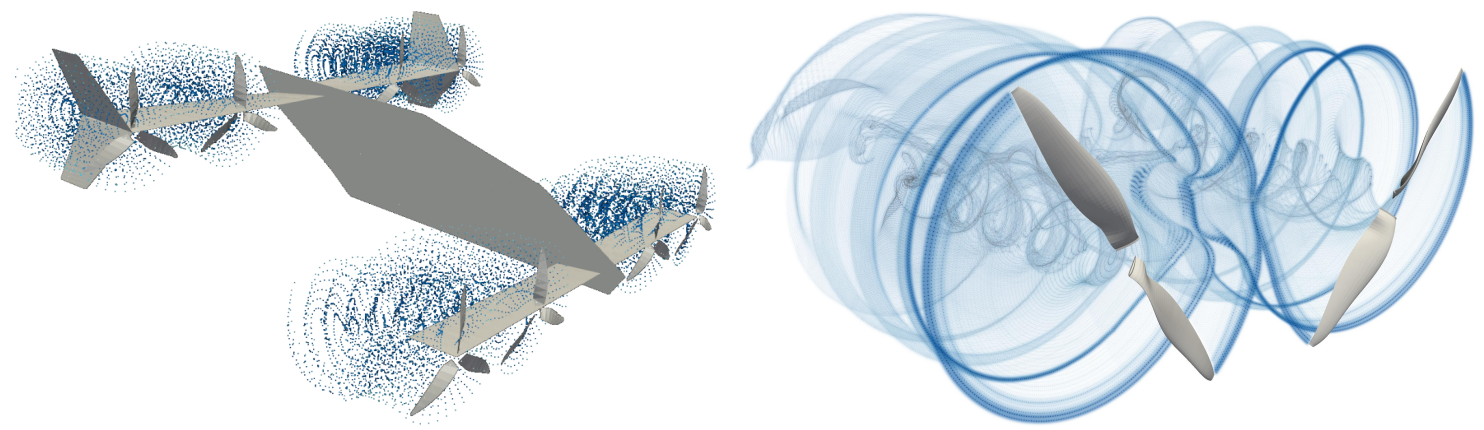

Fig. 3 Mid-fidelity VPM simulation of eVTOL aircraft (left) and high-fidelity VPM simulation of multirotor wake mixing [30] (right).

*In the author's experience, a RANS simulation of two side-by-side rotors in STAR-CCM+ requires about 1000 processor hours. Meanwhile, a VPM simulation with FLOWVPM, at a comparable resolution, requires only 3 processor hours. 
a VPM-based multirotor model and present extensive validation across the flight envelope. A summary of VPM theory and the numerical schemes used in this study are presented in Section II. The multirotor model is introduced in Section III along with a convergence study and recommendations for numerical stability and computational efficiency. In Sections IV.B and IV.C, validation of the individual rotor is presented in both hovering and forward-flight configurations at low, moderate, and high Reynolds numbers. Validation of the hovering multirotor is presented in Section IV.D, comparing predictions to experimental measurements of thrust drop and unsteady loading associated with rotor-on-rotor interactions.

Alongside the multirotor model validation, this paper presents the following contributions to the viscous vortex particle method:

- A simplistic, efficient, and accurate approach for the computation of vortex stretching through a complex-step derivative approximation embedded in the fast multipole method (Section II.E).

- A procedure for wake trimming, achieving numerically-stable hover simulations that avoid the instabilities arising in the wake from under-resolved length scales after turbulent breakdown (Section III.B).

- Decoupling of temporal and spatial resolutions encountered in VPM-based rotor simulations (Section III.C). We also present a detailed convergence study on four parameters characterizing temporal and spatial resolutions that is used for quantification of numerical error (Section III.D).

\section{Viscous Vortex Particle Method}

By a way of introduction to the viscous vortex particle method, Sections II.A and II.B present a concise development of the equations governing the method. For a more involved description, the reader is referred to the literature [31, 66]. As part of this study, a VPM code was developed for the simulation of flight vehicles and design optimization, called FLOWVPM (FLight, Optimization, and Wind VPM). Sections II.C through II.F describe the numerical schemes implemented in FLOWVPM.

\section{II.A Governing Equations}

The Navier-Stokes equations of an incompressible flow can be formulated into its vorticity form by taking the curl over the momentum equation

$$
\nabla \times\left(\frac{\partial \mathbf{u}}{\partial t}+(\mathbf{u} \cdot \nabla) \mathbf{u}\right)=\nabla \times\left(-\frac{1}{\rho} \nabla p+v \nabla^{2} \mathbf{u}\right),
$$

leading to an expression that is not dependent on the pressure field:

$$
\frac{D \omega}{D t}=(\omega \cdot \nabla) \mathbf{u}+v \nabla^{2} \omega .
$$

The material derivative expressed in Eq. (1), and the material-conservative nature of the vorticity makes the $\omega$ field especially fit for a Lagrangian description. In order to do so, the field is discretized into Lagrangian elements (called vortex particles) using a radial basis function approximation of basis $\zeta_{\sigma}$ and coefficients $\boldsymbol{\Gamma}_{p}$ :

$$
\omega(\mathbf{x}, t) \approx \sum_{p} \boldsymbol{\Gamma}_{p}(t) \zeta_{\sigma}\left(\mathbf{x}-\mathbf{x}_{p}(t)\right)
$$

Each particle represents a volume of fluid that travels with the local velocity as in Eq. (3), where $\mathbf{x}_{p}$ is the position of the $p$-th particle. The coefficient $\boldsymbol{\Gamma}_{p}$, termed vectorial circulation or vortex strength, is defined as $\boldsymbol{\Gamma}_{p} \equiv \int_{\operatorname{vol}_{p}} \omega \mathrm{d} V$ and approximated as $\Gamma_{p} \approx \omega_{p} \operatorname{vol}_{p}$, where $\omega_{p}$ is the vorticity associated to the $p$-th particle of volume vol $p$. Applying this particle discretization to Eq. (1), it is obtained that the vectorial circulation of each particle evolves as in Eq. (4), where the first right-hand-side term introduces vortex stretching, while the second represents a scheme for modeling the viscous diffusion $v \nabla^{2} \omega$. Thus, Eqs. (3) and (4) are the governing equations of the evolution of vorticity-governed flow in a Lagrangian scheme.

$$
\begin{aligned}
& \frac{\mathrm{d}}{\mathrm{d} t} \mathbf{x}_{p}(t)=\mathbf{u}\left(\mathbf{x}_{p}(t), t\right) \\
& \frac{\mathrm{d}}{\mathrm{d} t} \boldsymbol{\Gamma}_{p}(t)=\left(\boldsymbol{\Gamma}_{p}(t) \cdot \nabla\right) \mathbf{u}\left(\mathbf{x}_{p}(t), t\right)+\left.\frac{\mathrm{d}}{\mathrm{d} t} \boldsymbol{\Gamma}_{p}(t)\right|_{\mathrm{visc}}
\end{aligned}
$$




\section{II.B Velocity Kernel}

By the Helmholtz Decomposition Theorem, an incompressible velocity field $\mathbf{u}$ dominated by vorticity is described by some vector potential field $\psi$ as $\mathbf{u}(\mathbf{x}, t)=\nabla \times \psi(\mathbf{x}, t)$. From the definition of the vorticity field, $\omega=\nabla \times \mathbf{u}$, we obtain the three-dimensional unbounded Poisson's problem

$$
\nabla^{2} \psi=-\omega
$$

which can also be rewritten using the particle approximation from Eq. (2) as

$$
\nabla^{2} \psi(\mathbf{x}) \approx-\sum_{p} \boldsymbol{\Gamma}_{p} \zeta_{\sigma}\left(\mathbf{x}-\mathbf{x}_{p}\right)
$$

The Poisson's problem yields that the velocity induced by the field of vortex particles is calculated as

$$
\mathbf{u}(\mathbf{x})=\sum_{p} g_{\sigma}\left(\mathbf{x}-\mathbf{x}_{p}\right) \mathbf{K}\left(\mathbf{x}-\mathbf{x}_{p}\right) \times \mathbf{\Gamma}_{p},
$$

where $g_{\sigma}$ is a normalized smoothing function derived from the chosen basis function $\zeta_{\sigma}$, and $\mathbf{K}(\mathbf{x})=-\frac{\mathbf{x}}{4 \pi\|\mathbf{x}\|^{3}}$ is the singular Newtonian kernel resulting from the three-dimensional Green's function of the unbounded Poisson's problem.

\section{II.C Kernel Regularization}

The velocity kernel shown in Eq. (5) involves the Newtonian kernel with norm $\|\mathbf{K}(r)\|=\frac{1}{4 \pi r^{2}}$, introducing a singularity as $r \rightarrow 0$. In order to regularize this singularity, the vortex particle is defined as a vortex "blob" with a smoothing radius $\sigma$ that forces its influence to decay inside this radius. This is achieved by choosing a basis $\zeta_{\sigma}$ that will end up generating a smoothing function $g_{\sigma}$ that decays faster than the growth of the singular term, leading to a regularized kernel $\mathbf{K}_{\sigma}(\mathbf{x})=g_{\sigma}(\mathbf{x}) \mathbf{K}(\mathbf{x})$.

Multiple kernels have been proposed in the literature meeting different algebraic properties and computational complexity [31]. A Gaussian-based kernel simplifies the calculation of higher derivatives due to the recursive behavior of the exponential, and enables the use of the core spreading viscous scheme (explained in the next section). Thus, in FLOWVPM, we implement the Gaussian basis function $\zeta_{\sigma}(\mathbf{x})=\frac{1}{\sigma^{3}} \zeta\left(\frac{\|\mathbf{x}\|}{\sigma}\right)$ with $\zeta(r)=\frac{1}{(2 \pi)^{3 / 2}} \exp \left(-\frac{r^{2}}{2}\right)$, leading to

$$
g_{\sigma}(\mathbf{x})=\operatorname{erf}\left(\sqrt{\frac{\|\mathbf{x}\|^{2}}{2 \sigma^{2}}}\right)-\sqrt{\frac{4}{\pi}} \sqrt{\frac{\|\mathbf{x}\|^{2}}{2 \sigma^{2}}} \exp \left(-\frac{\|\mathbf{x}\|^{2}}{2 \sigma^{2}}\right)
$$

\section{II.D Fast Multipole Acceleration}

The governing equations-Eqs. (3) and (4) - coupled with the velocity kernel in Eq. (5) require the calculation of all particle interactions, posing a $N$-body problem with computational complexity $O\left(N^{2}\right)$, where $N$ is the number of particles. This complexity is prohibitive as the number of particles readily escalates to the order of the hundreds of thousands in mid-fidelity simulations. An alternative to the direct calculation of particle interactions is the clustering of particles and approximation of direct interactions between well-separated particles with the interactions between clusters. A popular and simple implementation of this concept is the Treecode method [67] that reduces the computational effort to $O(N \log (N))$. An even more efficient implementation is the fast multipole method (FMM) introduced by Greengard and Rokhlin [68-70]. Selected as one of the top ten algorithms of the 20th century, FMM reduces the original quadratic complexity to a linear problem, $O(N)$, while controling the error of the approximation down to any arbitrary precision. However, this method is often avoided due to its considerable conceptual and implementation intricacy.

In this study, FMM is applied in the computation of velocity and vortex stretching using a modified version of the open-source code ExaFMM developed by Barba and Yokota [46, 71, 72]. Fig. 4 shows the wall-clock time during the computation of velocity and vortex stretching through the FMM, evidencing linear scaling up to problems with millions of particles. The calculation of higher derivates for vortex stretching is explained in the following section. 


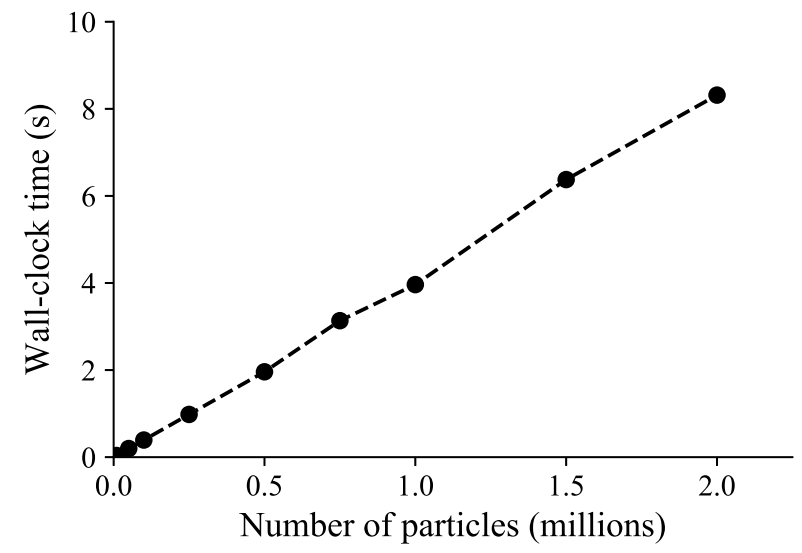

Fig. 4 Wall-clock time of velocity and vortex stretching computation, showing the linear scaling achieved through the FMM.

\section{II.E Vortex Stretching Through Complex-step Derivative Approximation}

The classic FMM [70] was originally formulated to calculate interactions of the form $1 / r$, as is the vectorial potential $\psi$. However, we are interested in determining the vorticity-induced velocity $\mathbf{u}$ and vortex stretching $(\boldsymbol{\Gamma} \cdot \nabla) \mathbf{u}$ rather than the vector potential $\psi$ calculated by the classic FMM. These fields can be obtained from the derivatives of $\psi$ as

$$
\mathbf{u}=\nabla \times \psi
$$

and

$$
(\boldsymbol{\Gamma} \cdot \nabla) \mathbf{u}=\left[\begin{array}{ccc}
\frac{\partial^{2} \psi_{3}}{\partial x_{1} \partial x_{2}}-\frac{\partial^{2} \psi_{2}}{\partial x_{1} \partial x_{3}} & \frac{\partial^{2} \psi_{3}}{\partial x^{2}}-\frac{\partial^{2} \psi_{2}}{\partial x_{2} \partial x_{3}} & \frac{\partial^{2} \psi_{3}}{\partial x_{3} \partial x_{2}}-\frac{\partial^{2} \psi_{2}}{\partial x_{3}{ }^{2}} \\
\frac{\partial^{2} \psi_{1}}{\partial x_{1} \partial x_{3}}-\frac{\partial^{2} \psi_{3}}{\partial x_{1}{ }^{2}} & \frac{\partial^{2} \psi_{1}}{\partial x_{2} \partial x_{3}}-\frac{\partial^{2} \psi_{3}}{\partial x_{2} \partial x_{1}} & \frac{\partial^{2} \psi_{1}}{\partial x_{3}{ }^{2}}-\frac{\partial^{2} \psi_{3}}{\partial x_{3} \partial x_{1}} \\
\frac{\partial^{2} \psi_{2}}{\partial x_{1}{ }^{2}}-\frac{\partial^{2} \psi_{1}}{\partial x_{1} \partial x_{2}} & \frac{\partial^{2} \psi_{2}}{\partial x_{2} \partial x_{1}}-\frac{\partial^{2} \psi_{1}}{\partial x_{2}{ }^{2}} & \frac{\partial^{2} \psi_{2}}{\partial x_{3} \partial x_{1}}-\frac{\partial^{2} x_{1}}{\partial x_{3} \partial x_{2}}
\end{array}\right]\left[\begin{array}{c}
\Gamma_{1} \\
\Gamma_{2} \\
\Gamma_{3}
\end{array}\right] .
$$

Hence, the FMM implementation must also include the computation of first and second derivatives of $\psi$. For first-order derivatives, most authors derive and implement the analytical expression from local and multipole expansions [36]. However, for second-order derivatives, authors have differed between implementing an analytical calculation [45, 56], or computing the derivates through finite difference [36]. The former approach is carried out with exact precision, with the drawback of further complicating the FMM implementation. On the other hand, the latter approach offers simplicity while forfeiting numerical accuracy. For the work presented in this paper, we introduce a new approach: the calculation of vortex stretching through a complex-step derivative approximation (CSDA) [73]. This approach is conceptually simple and easy to implement, while achieving machine precision without increasing the computational cost.

Let the real-variable analytic function $f: \mathbb{R} \rightarrow \mathbb{R}$ be generalized to its complex-variable form $f^{*}: \mathbb{C} \rightarrow \mathbb{C}$, and $x_{0} \in \mathbb{R}$ an arbitrary point where we desire to evaluate the first derivative of $f$. Let $h \in \mathbb{R}$ be an arbitrarily small step, the function $f^{*}$ can be Taylor-expanded around $x_{0}+i h$ to obtain the derivative of $f$ as

$$
\frac{\mathrm{d} f}{\mathrm{~d} x}\left(x_{0}\right)=\frac{\operatorname{Im}\left(f^{*}\left(x_{0}+i h\right)\right)}{h}+O\left(h^{2}\right) .
$$

Let us now choose an $h$ sufficiently small—say $h=10^{-30}$ for double floating point precision—, and we have obtained a numerical approximation of the derivative of $f$ that is as accurate as machine precision.

CSDA can be directly applied to any real-variable real-valued function $f: \mathbb{R} \rightarrow \mathbb{R}$ by generalizing the function to the complex plane $f^{*}: \mathbb{C} \rightarrow \mathbb{C}$ and using this plane for carrying on the numerical differentiation. However, multipole and local expansions of the FMM are computed through complex-valued functions $Y: \mathbb{R} \rightarrow \mathbb{C}$ already using the complex plane, requiring the formulation of CSDA in a multicomplex space. Hence, we will now define the multicomplex arithmetic needed for implementing CSDA into the FMM.

Let the complex set $\mathbb{C}^{1}$ with imaginary unit $i_{1}$ be $\mathbb{C}^{1}=\left\{x+i_{1} y / x, y \in \mathbb{R}\right\}$, and let us define the multicomplex set $\mathbb{C}^{2}$ with imaginary unit $i_{2}$ as $\mathbb{C}^{2}=\left\{z+i_{2} w / z, w \in \mathbb{C}^{1}\right\}$, any complex-valued analytical function $Y: \mathbb{R} \rightarrow \mathbb{C}$ will then 
be generalized into the multicomplex space $\mathbb{C}^{2}$ as $Y^{*}: \mathbb{C}^{1} \rightarrow \mathbb{C}^{2}$. In this generalization we have transformed the set $\mathbb{R}$ into $\mathbb{C}^{1}$, and $\mathbb{C}$ into $\mathbb{C}^{2}$; meaning that a variable $x$ originally in $\mathbb{R}$ now becomes $x^{*}=x+i_{1} 0$, and a variable $z=a+i b$ originally in $\mathbb{C}$ now becomes $z^{*}=a^{*}+i_{2} b^{*}=\left(a+i_{1} 0\right)+i_{2}\left(b+i_{1} 0\right)=a+i_{2} b$. With this definition, the axis $i_{1}$ will carry the CSDA computation and is not intended to interact with the axis $i_{2}$-hence, the products $i_{1} i_{2}$ and $i_{2} i_{1}$ will purposefully remain undefined as to avoid any unintended arithmetic. The derivative of $Y$ is then computed as

$$
\frac{\mathrm{d} Y}{\mathrm{~d} x}(x) \approx \frac{\operatorname{Im}_{1}\left(Y^{*}\left(x+i_{1} h\right)\right)}{h} .
$$

Since multipole and local expansion are computed through spherical harmonic functions $Y_{n}^{m}: \mathbb{R}^{2} \rightarrow \mathbb{C}$ of the form

$$
Y_{n}^{m}(\theta, \phi)=\sqrt{\frac{(n-|m|) !}{(n+|m|) !}} \cdot P_{n}^{|m|}(\cos \theta) e^{i m \phi},
$$

where $P_{n}^{|m|}$ are the associated Legendre functions, we now show how this multicomplex transformation applies to the complex exponential and complex product in Eq. (6). Let the complex-valued function $Y: \mathbb{R} \rightarrow \mathbb{C}$ be defined as $Y(x)=e^{i x}$, the derivative is easily determined analytically as $\frac{\mathrm{d} Y}{\mathrm{~d} x}(x)=i e^{i x}$. On the other hand, its multicomplex generalization $Y^{*}: \mathbb{C}^{1} \rightarrow \mathbb{C}^{2}$ is defined as

$$
Y^{*}(z)=e^{i_{2} z}
$$

and the derivative $\frac{\mathrm{d} Y}{\mathrm{~d} x}(x)$ is computed through CSDA as

$$
\begin{aligned}
\frac{\mathrm{d} Y}{\mathrm{~d} x}(x) & \approx \frac{\operatorname{Im}_{1}\left(Y^{*}\left(x+i_{1} h\right)\right)}{h} \\
& =\frac{1}{h} \operatorname{Im}_{1}\left(e^{i_{2} x}\left(\cos i_{2} h+i_{1} \sin i_{2} h\right)\right) \\
& =i_{2} e^{i_{2} x} \frac{\sinh h}{h} \quad, \text { where } \lim _{h \rightarrow 0} \frac{\sinh h}{h}=1 \\
& \stackrel{h \rightarrow 0}{=} i_{2} e^{i_{2} x},
\end{aligned}
$$

obtaining the same result than the analytical derivation in the limit $h \rightarrow 0$.

Now we show how CSDA is carried on the scalar-complex product. Let $Y: \mathbb{R} \rightarrow \mathbb{C}$ be defined as $Y(x)=c x=$ $\left(c_{1}+i c_{2}\right) x$ with $c_{1}, c_{2} \in \mathbb{R}$, the derivative is trivially $\frac{\mathrm{d} Y}{\mathrm{~d} x}(x)=c$. Defining its multicomplex generalization $Y^{*}: \mathbb{C}^{1} \rightarrow \mathbb{C}^{2}$ as

$$
Y^{*}(z)=c^{*} z
$$

with $c^{*} \in \mathbb{C}^{2} / c^{*}=c_{1}^{*}+i_{2} c_{2}^{*}=\left(c_{1}+i_{1} 0\right)+i_{2}\left(c_{2}+i_{1} 0\right)$, the derivative $\frac{\mathrm{d} Y}{\mathrm{~d} x}(x)$ is then computed through CSDA as

$$
\begin{aligned}
\frac{\mathrm{d} Y}{\mathrm{~d} x}(x) & \approx \frac{\operatorname{Im}_{1}\left(Y^{*}\left(x+i_{1} h\right)\right)}{h} \\
& =\frac{1}{h} \operatorname{Im}_{1}\left(\left(c_{1}+i_{2} c_{2}\right)\left(x+i_{1} h\right)\right) \\
& =\frac{1}{h}\left(c_{1}+i_{2} c_{2}\right) h \\
& =c^{*} .
\end{aligned}
$$

The complex exponential and the complex product are not only good example cases of multicomplex arithmetic, but they are also the only two multicomplex operations needed for carrying the CSDA through multipole and local expansions of the FMM.

In order to compare accuracy between finite difference and CSDA, vortex stretching was computed through direct calculation, FMM with finite difference, and FMM with CSDA in 1000 randomly-generated particles. The error relative to the direct calculation at each particle is shown in Fig. 5, evidencing the subtractive and round-off error introduced by the finite difference, meanwhile CSDA approaches floating-point precision. Furthermore, the CSDA is applied only in the local-to-particle translation step of the FMM, which takes less than $2 \%$ of the computational time of the overall FMM algorithm [74]. Thus, vortex stretching calculated through CSDA increases the computational cost only marginally compared to the analytical calculation, while offering a much simpler implementation. 


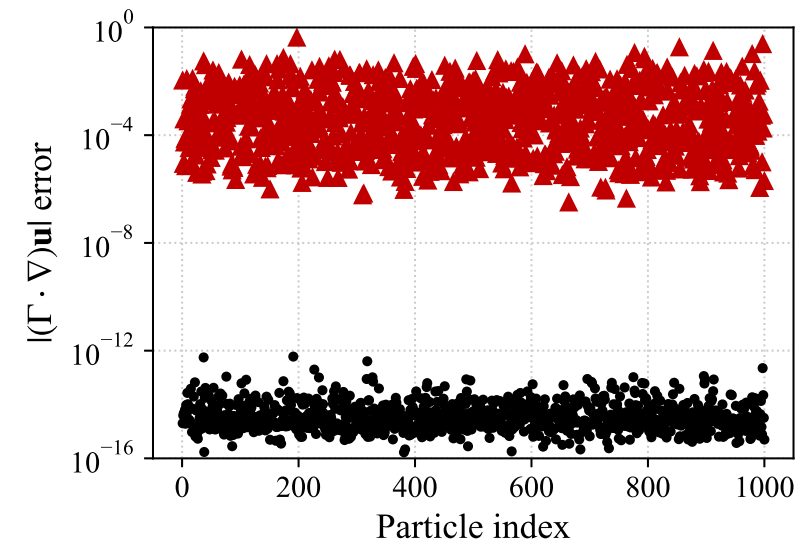

Fig. 5 Error of vortex stretching computed through finite difference (red markers) and CSDA (black markers).

\section{II.F Viscous Diffusion and Other Schemes}

In this study, the viscous diffusion term in Eq. (4) is solved through the core spreading method [75] coupled with the radial basis function (RBF) interpolation approach developed by Barba [32, 76, 77], which avoids the need for particle splitting while allowing the viscous VPM to be a truly meshless method. In the core spreading method, a Gaussian radial basis allows the viscous diffusion term in Eq. (1) to be solved by spreading the smoothing radius as

$$
\frac{\mathrm{d} \sigma^{2}}{\mathrm{~d} t}=2 v
$$

Core sizes $\sigma$ are reset to their initial value after they have overgrown, while new vectorial circulations $\boldsymbol{\Gamma}_{p}$ are calculated through an RBF interpolation preserving the vorticity field $\omega$.

In FLOWVPM, vortex stretching is solved in the transposed scheme as explained in Winckelmann's thesis [66], and the divergence of the vorticity field is treated through the relaxation scheme developed by Pedrizzeti [78]. The time integration of the governing equations is done through a low-storage third-order Runge-Kutta scheme [79].

\section{VPM-based Rotor Simulation}

\section{III.A Propeller Model}

The propeller model developed for this study is formulated in the same numerical scheme of the VPM, capturing unsteady dynamics and viscous effects while benefiting from the computational speed and linear scaling of the VPM. Each rotor blade is modeled as a rotary lifting surface, and the physics of interest are broken down into three aspects: load distribution, blade-induced velocity, and wake-induced velocity. The load distribution is calculated using blade elements, and is used to derive the circulation along the lifting surface. In turn, the blade-induced velocity is obtained from the circulation distribution by embedding vortex particles along the surface that preserve such circulation, as shown in Fig. 6. As the blade moves, vorticity is shed off the trailing edge as free vortex particles stemming from both unsteady loading and trailing circulation. Thus, blade and wake-induced velocities are all computed through the FMM, achieving an efficient scaling of the simulation to the order of millions of particles, while all unsteady dynamics are resolved as the VPM steps in time. Fig. 7 shows the computation diagram of the simulation.

In the setup stage (blue block in Fig. 7), the user specifies the rotor geometry and blades are discretized into elements. Two-dimensional aerodynamic characteristics of each blade element are precomputed through XFOIL at the corresponding local Reynolds number (considering rotational and freestream velocity), thus capturing viscous effects. A Prandtl-Glauert compressibility correction is applied to lift curves, capturing compressibility effects. Both lift and drag curves are then treated to capture three-dimensional drag and stall-delay effects encountered in rotor blades [80] and the Viterna method [81] is applied to obtain post-stall $\pm 180^{\circ}$ extrapolations of these curves.

In the first time step of the simulation, the effective angle of attack (AOA) of every element is calculated from the freestream and local rotational velocity. The effective AOA is then used to calculate the load distribution from the 
precalculated airfoil lift and drag curves. In all subsequent time steps, the propellers are rotated and vortex particles are shed from trailing edges. The Navier-Stokes equations are solved in their vorticity form (Eq. (1)) as convection, stretching, and viscous diffusion terms (Eqs. (3) and (4)) are calculated at the position of each vortex particle. The velocity induced by the wake and lifting surfaces is then added to the freestream and rotational velocities in order to calculate the new effective angle of attack and dynamic pressure, and the load distribution is updated (gray block in Fig. 7). The process is then iterated until the end of the simulation, $t_{f}$, as shown in the green block. This algorithm is analogous to the method developed by Jo and Lee [51, 82], except that we omit the minor iterations and let load distributions converge in time as the wake is deployed. Also, we model all lifting surfaces using embedded vortex particles in order to perform all computation through the VPM, which allows us to efficiently scale the simulation in order $O(N)$. Fig. 8 shows the simulation of two side-by-side rotors in hover, exemplifying the unsteady dynamics and wake mixing achieved with this propeller model.

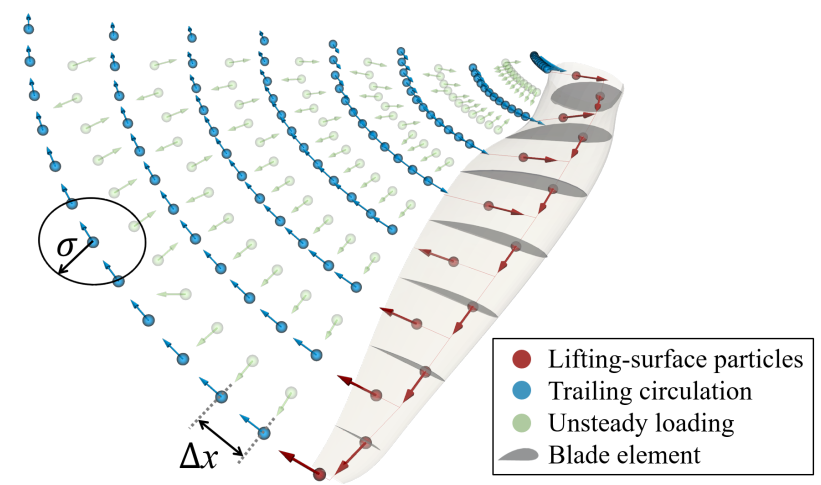

Fig. 6 Blade and wake deployment in the propeller model. Particles are colored by their respective source of vorticity; arrows indicate the direction of vortex strength $\Gamma_{p}$.

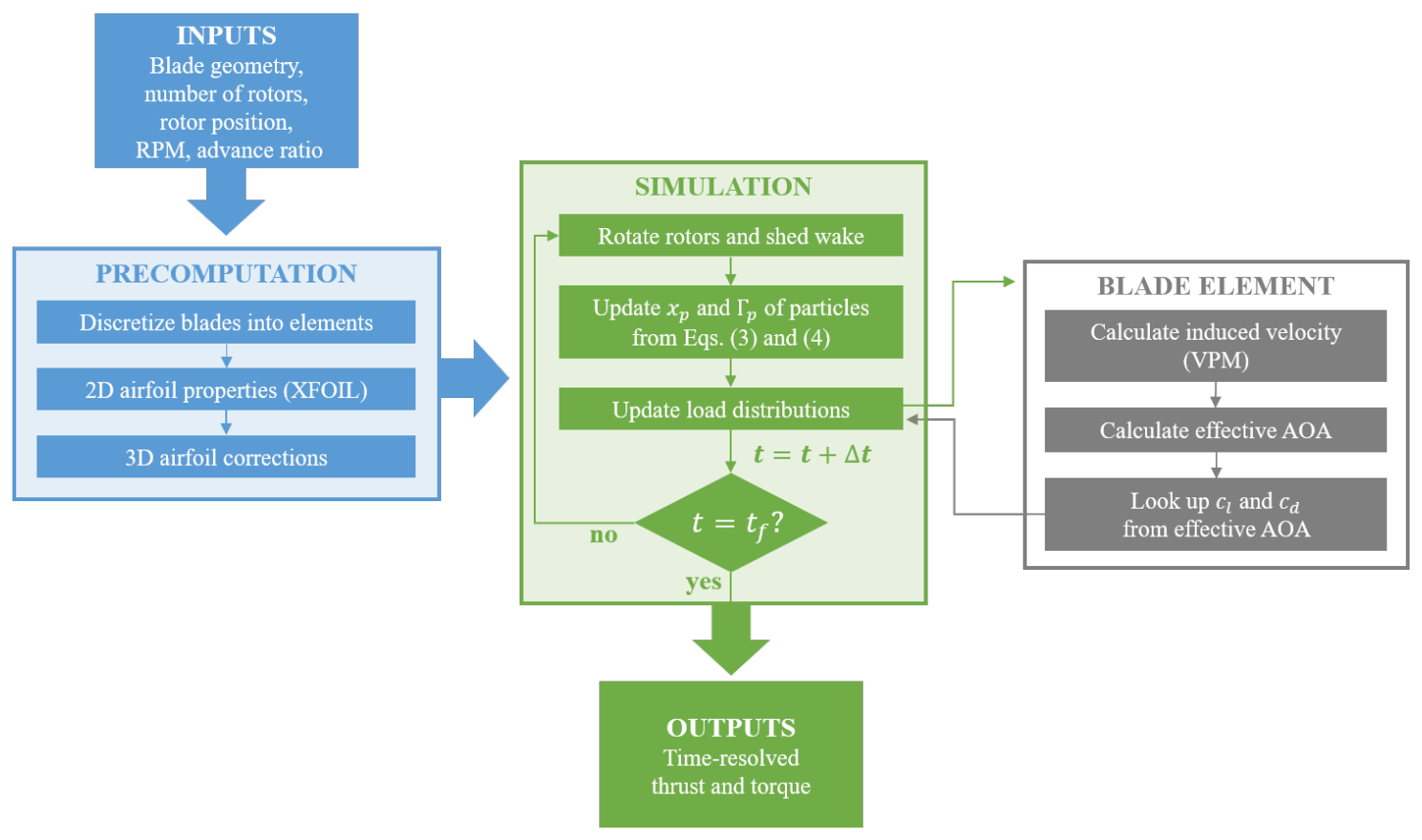

Fig. 7 Computation diagram of single and multi-rotor simulation. 


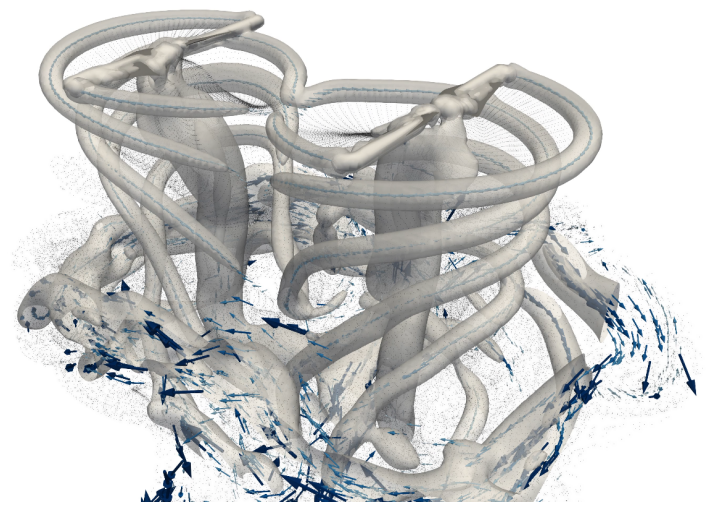

Fig. 8 Simulation of hovering multirotor, with particles and vortex strengths shown as dots and arrows respectively, superimposed with isosurfaces of the vorticity field.

\section{III.B Wake Trimming Achieving Stable Simulations}

The main strength of the VPM lies in its low numerical dissipation enabling accurate preservation of vortical structures at a low computational cost. For instance, Fig. 9 shows the wake resulting from a coarse simulation, demonstrating that VPM is able to characterize the evolution of the wake-from near to far field and turbulent breakdown capturing all mechanisms of transition (i.e., short-wave instabilities, leapfrogging, and vortex pairing [83, 84])—even with a coarse spatial resolution. However, a low numerical dissipation also means that some numerical instabilities may not be properly damped. The VPM is a direct-numerical simulation approach, meaning that it does not use a turbulence model but rather resolves all length scales down to the spatial resolution of the simulation. Since VPM is free of the artificial viscosity introduced by numerical dissipation in mesh-based CFD, we have observed that the unresolved length scales give rise to numerical instabilities that require an increased spatial resolution to ensure stability after turbulent breakdown. As an example, Fig. 10 shows the history of thrust coefficient $C_{T}$ in a hover simulation (red markers). During the first three revolutions, $C_{T}$ converges to a steady value once the near field has fully developed. However, as the wake transitions from far field to breakdown regime, numerical instabilities start to arise at the head of the wake, leading to numerical blowup after nine revolutions. We have observed that the turbulent breakdown regime can be further resolved by increasing the spatial resolution, achieving a longer simulation at the tradeoff of an ever-increasing computational cost.

In order to bring about numerical stability within a feasible computational cost, the wake is trimmed off as it moves further into the turbulent breakdown regime. This is done by defining a downstream threshold after which particles in the wake are removed. At the beginning of the simulation, such threshold is defined close to the plane of rotation since the wake tends to breakdown while is being deployed. As the simulation advances, the threshold is progressively moved further away until the wake becomes fully developed. As an example, Fig. 11 shows the simulation of a rotor in hover where the wake is being trimmed at six and ten revolutions, moving the threshold away from the plane of rotation. Fig. 10 shows in blue the history of $C_{T}$ with wake trimming at six, ten, fourteen, and seventeen revolutions. During the early stages of the simulation, trimming tends to perturb the near field as noted by the jumps in $C_{T}$. However, as the

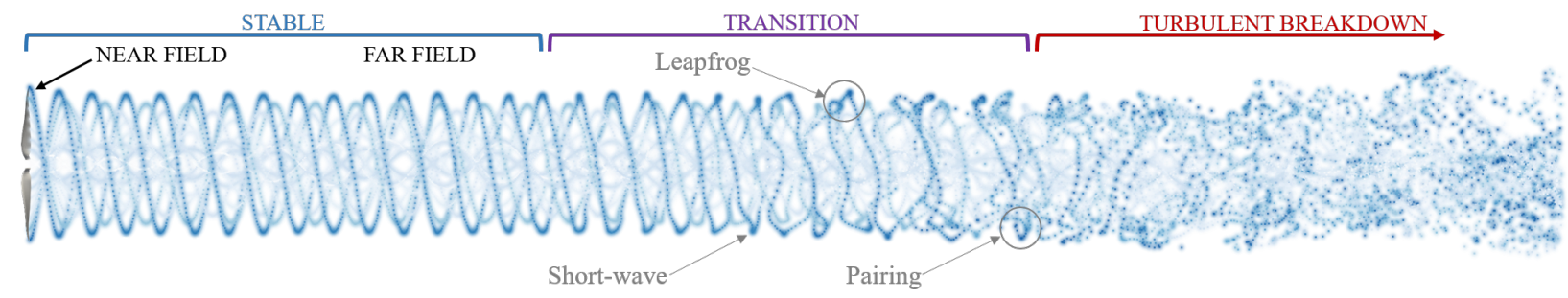

Fig. 9 Simulation of propeller at $J=0.35$ showing evolution of wake from near to far field and transition to turbulent breakdown. 


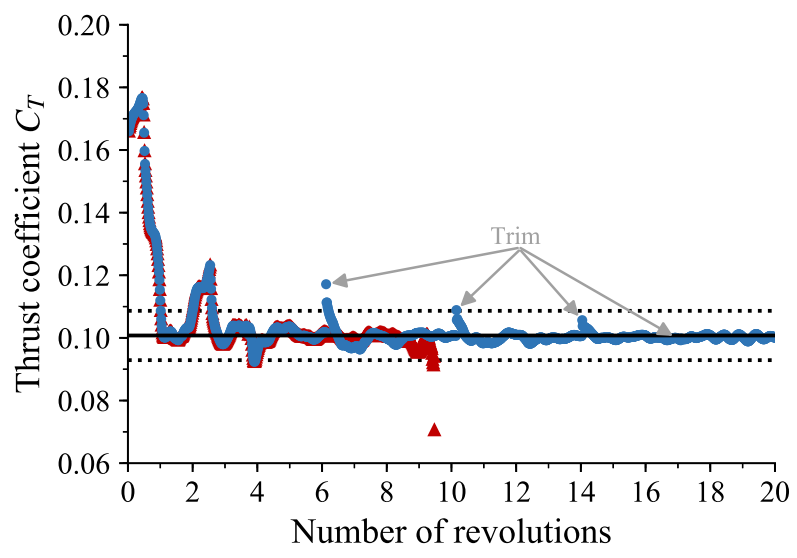

Fig. 10 History of thrust coefficient $C_{T}$ in hovering single-rotor simulation with and without wake trimming (blue and red markers, respectively). Solid black line shows experimental mean thrust and dashed lines enclose the standard deviation as reported in Zhou et al. [20] and Ning [21].
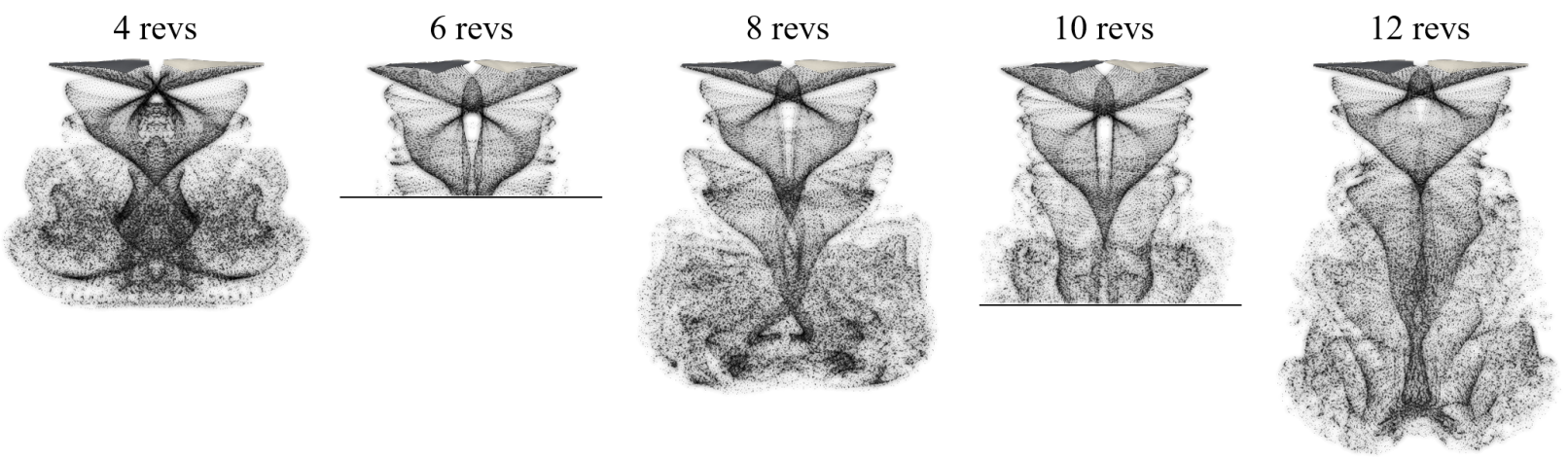

Fig. 11 Evolution of wake of rotor in hover with wake trimming at 6 and 10 revolutions.

wake develops and the threshold is moved further away, the trimming has less of an effect on the near field and the jump eventually disappears once the wake becomes fully developed. Thus, with this trimming procedure, an indefinitely-long simulation is obtained. For hover simulations, we have observed that the trimming threshold can be set as far as one diameter downstream into the turbulent breakdown region, obtaining a completely stable simulation while still capturing all the dynamics of vortex breakdown.

\section{III.C Decoupled Temporal and Spatial Resolutions}

In VPM-based rotor simulations, spatial resolution is dictated simultaneously by the particle density and the smoothing radius $\sigma$, with the former giving the spatial discretization, and the latter giving the smallest resolved length scale. Studies have pointed out that numerical stability is conditioned to a spatial discretization smaller than the smoothing radius (discussed in the next section), meaning that as the smoothing radius is decreased, the frequency that particles are shed off the blade must in turn be increased to obtain a larger particle density. A common practice in VPM-based simulations is to shed particles at every time step, inevitably coupling temporal and spatial resolutions: if the shedding of particles is controlled by the time stepping, a finer spatial resolution is only achieved by also increasing the temporal resolution. This coupling not only increases the computational cost unnecessarily, but it also embroils any convergence study as the numerical error will not decrease monotonically with the coupled spatio-temporal resolution.

One approach to control the spatial resolution independently of temporal resolution is to generate a background mesh that projects and resets all particle positions after convection. This approach, called the vortex particle-mesh or vortex-in-cell method $[85,86]$, also provides the mechanisms for LES turbulence filtering and control of Lagrangian distortion [41, 43]. However, the introduction of a background mesh forfeits some of the benefits of a purely Lagrangian, 
meshless simulation.

In order to decouple temporal and spatial resolutions without a mesh, we introduce a procedure for the shedding of particles independent of time stepping. Let $N_{\text {steps }}$ and $N_{\text {sheds }}$ be the number of time steps and particle sheds in one rotor revolution, we impose $N_{\text {steps }}$ and $N_{\text {sheds }}$ to be multiples of each other. Let $m \in \mathbb{Z}^{+}$be such multiple. If the target number of time steps is larger or equal to the number of sheds ( $\left.N_{\text {steps }} \geq N_{\text {sheds }}\right)$, particles are simply shed every $m$ time steps as shown in Fig. 12a (the case $N_{\text {steps }}>N_{\text {sheds }}$ is not shown as it is analogous to the case $N_{\text {steps }}=N_{\text {sheds }}$ ). If the number of sheds is to be increased independently of time stepping, even becoming larger than the number of time steps $\left(N_{\text {steps }}<N_{\text {sheds }}\right.$ ), particles are shed $m$ times in every time step, as shown in Fig. $12 \mathrm{~b}$ (where $m=4$ ). Thus, the frequency of particles sheds can be controlled independently of the time stepping. However, this procedure may lead to a large density of particles towards the slower-moving parts of the blade, as observed in Fig. 12b. This poses no numerical difficulties, but it increases the computational cost unnecessarily.

In order to ease computational cost, now we introduce an improvement to the procedure that makes this decoupling more efficient. This is done by varying the number of particles that are shed along the blade, increasing the number of sheds only towards the faster-moving part of the blade. Let $f$ be the number of particles that are shed at a given blade element, $f$ is defined to increase with the radial position $r$ (ranging from 1 at the root, to $m$ at the tip) through the following function given as pseudo-code:

$$
f(r, m)=\max \left[1, \min \left[m, \text { floor }\left(\frac{1}{1-(r / R)}\right)\right]\right]
$$

Fig. 13 shows the distribution of particles shed along the blade as $m$ is increased. When applied to the simulation, the

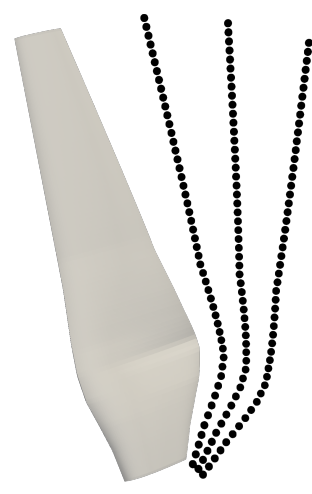

(a) $N_{\text {sheds }}=N_{\text {steps }}$

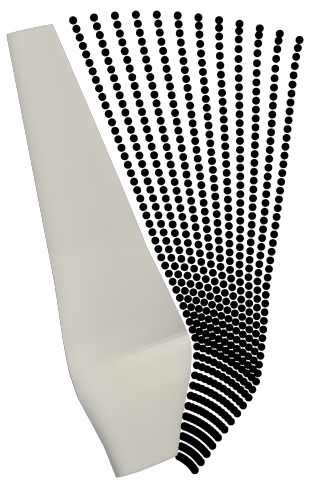

(b) $N_{\text {sheds }}=4 N_{\text {steps }}$

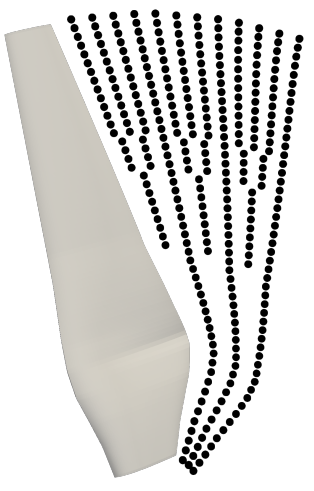

(c) $N_{\text {sheds }}=4 N_{\text {steps }}$

Fig. 12 Comparison of wake shedding cases after three time steps. (a) exemplifies the case $N_{\text {steps }} \geq N_{\text {sheds }}$ where particles are shed every $m$ steps ( $m=1$ is shown). (b) shows the $N_{\text {steps }}<N_{\text {sheds }}$ case that creates an excessive particle density towards the root, while (c) approximates a uniform particle density using Eq. (7).

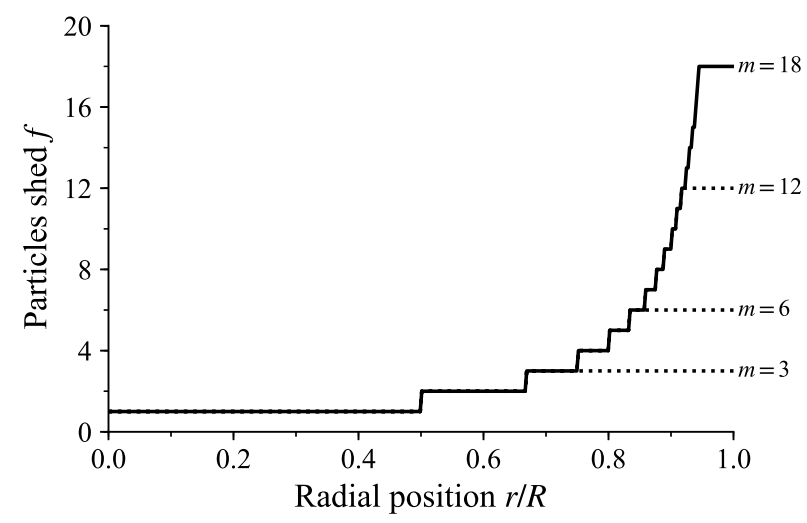

Fig. 13 Number of particles shed along blade as a function of $m$. 
particle field approaches a uniform density of particles as shown in Fig. 12c. With this decoupling of temporal and spatial resolutions, the convergence of the propeller simulation can be explored through well-defined, independent parameters as shown in the following section.

\section{III.D Convergence Study}

In order to quantify the convergence and numerical error associated with the propeller simulation, first we study the convergence of the vortex particle method by itself through the simulation of a vortex ring. The self-propelling vortex ring is a good VPM benchmarking case as is purely dominated by vorticity, and an analytical inviscid solution of its centroid velocity $U$ can be derived from the Navier-Stokes equations [87]. The analytical solution is $U=\frac{\Gamma}{4 \pi R}\left(\ln \left(\frac{8 R}{c}\right)-\beta\right)$, where $\Gamma$ is the circulation of the ring, $R$ is the ring's radius, $c$ is the toroidal core radius, and $\beta$ is a parameter dictated by the vorticity distribution along the toroid cross section. The algebraic kernel proposed by Winckelmans [31] is known to lead to $\beta=0.5$, hence in the simulation of the vortex ring we have used Winckelmans' kernel instead of the Gaussian kernel, with $\Gamma=1 \mathrm{~m}^{2} / \mathrm{s}, R=2 \mathrm{~m}$, and $c=0.002 R$. This corresponds to $U=0.310 \mathrm{~m} / \mathrm{s}$.

In the VPM simulation, the vortex ring is discretized into $N$ cross sections evenly spaced at a distance $\Delta x=2 \pi R / N$ along the toroid's centerline, with one particle at the centerline and one layer of particles around the circular cross section as explained in Ref. [66]. Making the smoothing radius $\sigma$ equal to the toroidal core radius $c$, the core overlap $\lambda$ between contiguous particles is defined as $\lambda \equiv \sigma / \Delta x$. Fig. 14 shows the convergence of centroid velocity to the analytical solution as the spatial resolution is increased from $N=25$ to $N=9425$. The figure in the right shows the numerical error in a semi-log scale as the core overlap is increased with $N$, evidencing that the error decreases exponentially after the threshold $\lambda=1$. This threshold has been identified in the literature as a necessary condition (i.e., $\lambda>1$ ) to construct a smooth vorticity field after particle discretization, ensuring stability and convergence of the VPM $[36,66,88]$, and is also equivalent to requiring the spatial discretization $\Delta x$ to be smaller than the smoothing radius $\sigma$. With this, it is shown that the numerical error of the VPM can be decreased down to any arbitrary precision.

In order to analyze the convergence of the propeller simulation, we have defined four independent parameters that determine temporal and spatial resolutions:

- $N_{\text {steps }}$, number of time steps per revolution.

- $N_{\text {sheds }}$, number of particle sheds per revolution.

- $f_{\sigma}$, particle smoothing factor defined as $f_{\sigma} \equiv \sigma / R$, with $R$ the rotor radius.

- $n$, number of blade elements per blade.

Within these parameters, $N_{\text {steps }}$ determines the temporal resolution of the simulation, $N_{\text {sheds }}$ determines the spatial discretization of the wake, $f_{\sigma}$ sets the smallest length scale resolved in the wake as it fixes the smoothing radius $\sigma$, and $n$ determines the spatial discretization of the blade. Both $f_{\sigma}$ and $N_{\text {sheds }}$ determine the core overlap $\lambda$ between tip particles, defined as $\lambda=\sigma / \Delta x=f_{\sigma} R / \Delta x$, where $\Delta x=2 \pi R / N_{\text {sheds }}$ is the spacing between particles shed from the blade tip as shown in Fig. 6 . Thus, for a given core overlap $\lambda$, the smoothing radius $\sigma$ decreases as particle density is increased. As already mentioned, the stability and convergence of the VPM requires meeting the condition of sufficient core overlap, $\lambda>1$, throughout the simulation. However, Lagrangian distortion will decrease the overlap once the
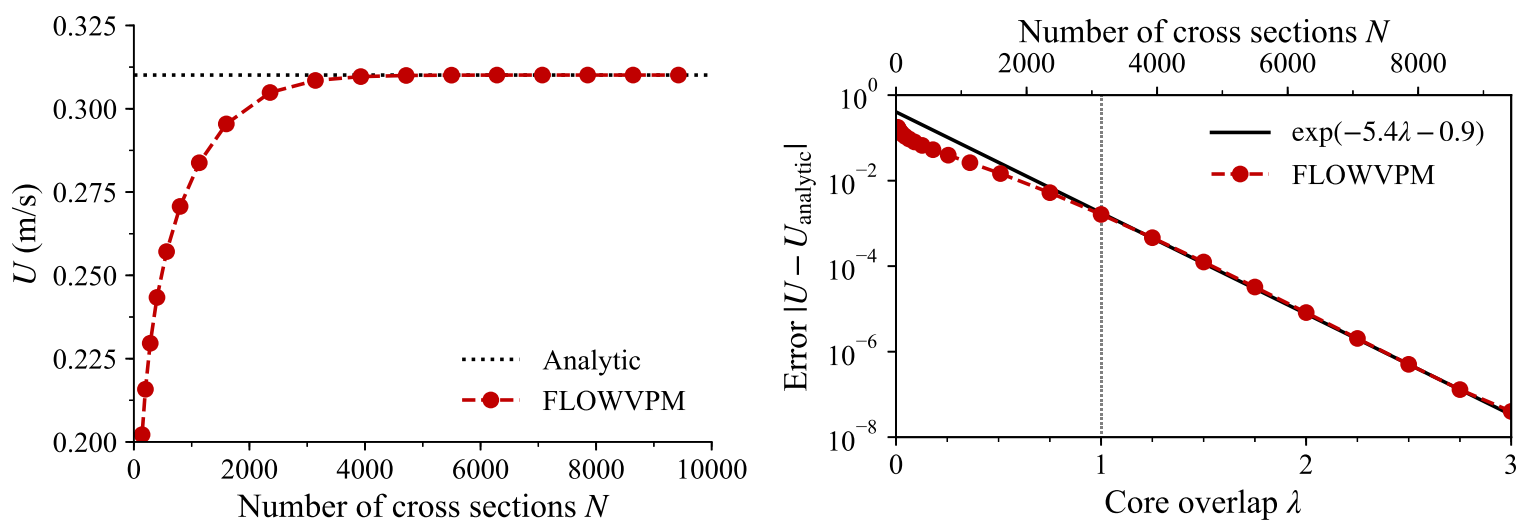

Fig. 14 Spatial convergence of the vortex-ring simulation to the analytical centroid velocity. 

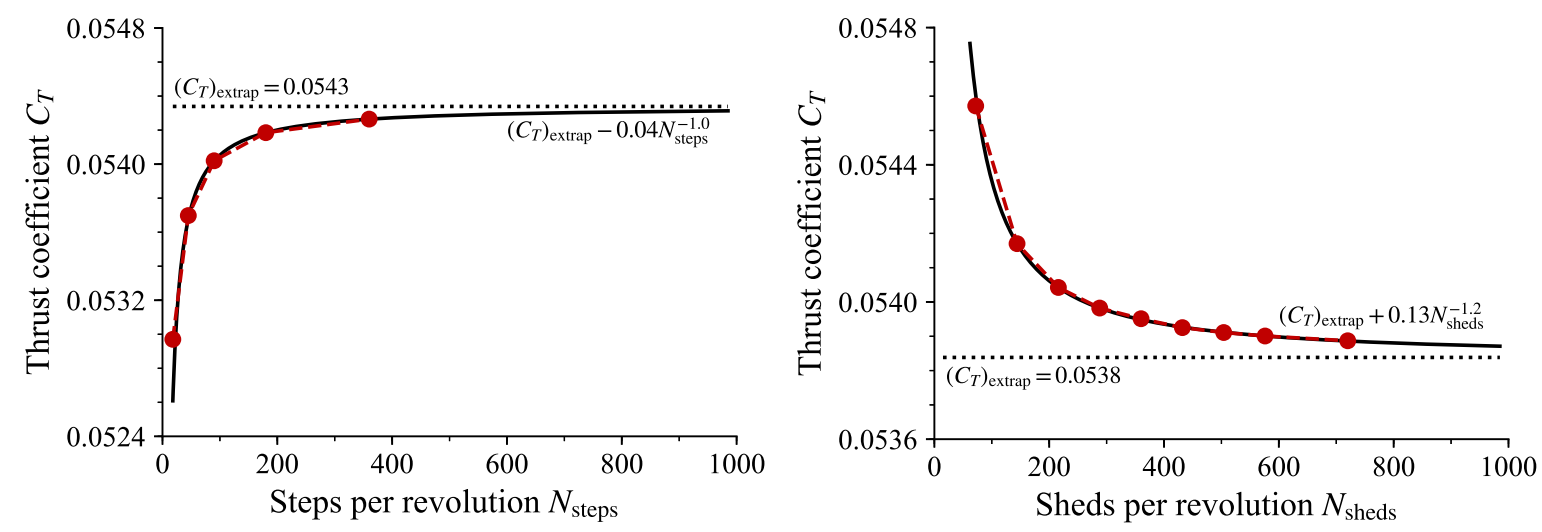

Fig. 15 Temporal (left) and spatial (right) convergence of the propeller simulation. Dotted lines correspond to Richardson extrapolations, $\left(C_{T}\right)_{\text {extrap }}$.

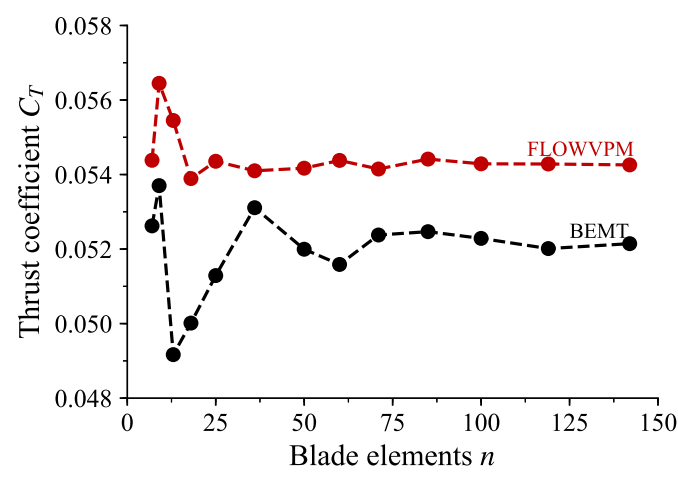

Fig. 16 Spatial convergence of propeller simulation with blade discretization, compared to a blade-element momentum theory (BEMT) code.

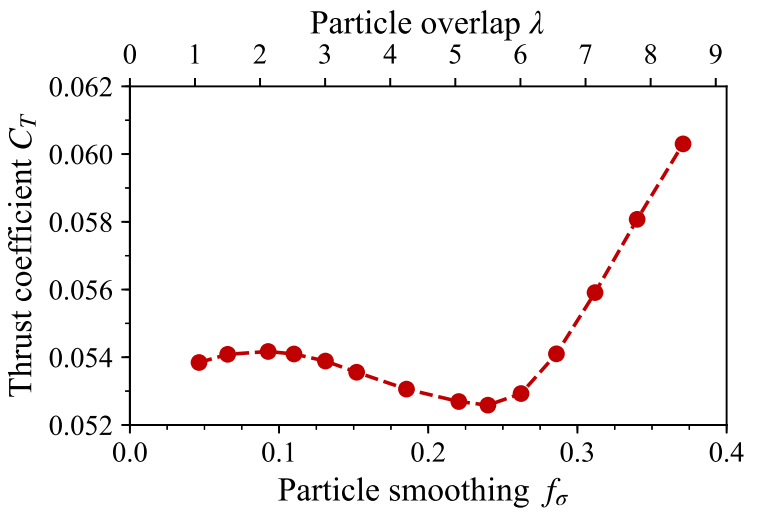

Fig. 17 Effects of particle smoothing on $C_{T}$ at a constant $N_{\text {sheds }}$ of 144.

wake starts to leapfrog and transition into turbulent breakdown. Our experience shows that $\lambda=2$ is the minimum tip-particle overlap achieving stable simulations up to turbulent breakdown from hover through high-advance-ratio configurations. To illustrate these parameters, Fig. 6 shows the first 12 time steps of a simulation with $N_{\text {steps }}=90$, $N_{\text {sheds }}=90, f_{\sigma}=0.07$ (corresponding to $\lambda=1.0$ ), and $n=7$.

The effects of each individual parameter on the numerical error was explored by simulating an APC 10x7 propeller at an advance ratio of 0.6 using the default values $N_{\text {steps }}=72, N_{\text {sheds }}=144, \lambda=2.125$ (or $f_{\sigma}=0.093$ ), and $n=50$ while each individual parameter was varied. The metric in this convergence study was the thrust coefficient $C_{T}$. Fig. 15 shows the convergence of $C_{T}$ as $N_{\text {steps }}$ is increased from $N_{\text {steps }}=18$ to $N_{\text {steps }}=360$ (corresponding to time steps of $20^{\circ}$ and $1^{\circ}$ of rotation, respectively) with its corresponding Richardson extrapolation, $\left(C_{T}\right)_{\text {extrap }}$. An approximation of the convergence order $p$ is obtained from a curve fit of the form $\left(C_{T}\right)_{\text {extrap }}+a(\Delta t)^{p}$ (shown in the figure as a solid line), where $\Delta t \propto 1 / N_{\text {steps. }}$. This results in $p=1.049$, indicating a first-order temporal convergence regardless of the third-order Runge-Kutta integration of the VPM's governing equations. The overall low-order convergence is believed to be due to performing an Euler step for the propeller rotation step. In spite of this, the simulation shows very low numerical error associated with temporal discretization, resulting an error relative to $\left(C_{T}\right)_{\text {extrap }}$ smaller than $1 \%$ for $N_{\text {steps }} \geq 72$ (or time steps smaller than $5^{\circ}$ ).

Fig. 15 shows the convergence of $C_{T}$ as $N_{\text {sheds }}$ is increased from $N_{\text {sheds }}=72$ to $N_{\text {sheds }}=576$ (corresponding to shedding particles every $5^{\circ}$ and $0.625^{\circ}$ of rotation, respectively) with its corresponding Richardson extrapolation, $\left(C_{T}\right)_{\text {extrap }}$. A curve fit of the form $\left(C_{T}\right)_{\text {extrap }}+b(\Delta x)^{p}$, where $\Delta x \propto 1 / N_{\text {sheds }}$, results in $p=1.204$ indicating approximate 
first-order spatial convergence. For $N_{\text {sheds }} \geq 144$, the error relative to $\left(C_{T}\right)_{\text {extrap }}$ becomes smaller than $1 \%$. Fig. 16 shows the convergence as the blade discretization $n$ is increased from $n=7$ to $n=142$, displaying a non-monotonic behavior. This is believed to be due to noise introduced in the splining and first-order interpolation process of airfoil lift and drag curves at every element along the blade. The same behavior can be observed in a code ${ }^{\dagger}$ using blade-element momentum theory (BEMT), as shown in the figure, which supports this belief. In the VPM-based simulation, $C_{T}$ varies by less than $0.3 \%$ with $n \geq 25$. Thus, with $n=50$ and $N_{\text {sheds }}=144$, the numerical error associated with spatial discretization is estimated to be smaller than $1 \%$.

Finally, Fig. 17 shows the effects of particle smoothing $f_{\sigma}$ and equivalent core overlap $\lambda$ on $C_{T}$. It is observed that $C_{T}$ starts to diverge as the stability threshold $(\lambda=1)$ is approached, while also diverging as $f_{\sigma}>0.25$ (equivalent to $\sigma$ larger than $0.25 R$ ) leads to unphysical wake dynamics caused by excessive smoothing. Since the instability associated with $\lambda \rightarrow 1$ plateaus at $\lambda \approx 2$, we have chosen $\lambda=2.125$ (or $f_{\sigma}=0.093$ ) for all subsequent simulations.

It must be noted that the convergence patterns shown in this section are obtained only after decoupling the temporal and spatial resolutions. Otherwise, a convergence study on the coupled spatio-temporal resolution leads to a numerical error that does not decrease monotonically, giving the false impression that the VPM is not a convergent method. Also, the aforementioned low discretization errors are only valid for the $C_{T}$ parameter and high advance ratio that was tested. We expect that flow field parameters like slipstream velocities would show a slower convergence and different sensitivities.

\section{Validation}

\section{IV.A Test Cases}

In this study we present validation of the VPM-based propeller model in both hover and forward-flight multirotor configuration at low, moderate, and high Reynolds numbers. Three experimental studies from the literature are used to validate the different operation configurations, using the three rotor geometries shown in Fig. 18 and summarized in Table 1. The hover case uses a rotor resembling the geometry of the DJI Phantom II quadcopter rotor, as described by Zhou et al. [20] and Ning [21], and the simulation of two side-by-side hovering rotors is compared to reported particle image velocimetry (PIV) and load cell measurements during low-Reynolds-number operation [20]. This rotor is $240 \mathrm{~mm}$ in diameter, and its geometry approximates the optimum rotor design obtained by imposing a constant effective angle of attack along the blade. The geometry was digitized assuming the twist distribution $\theta(r)=5.3^{\circ}+6.3^{\circ} \frac{R}{r}$ (with $r$ the radial position) shown in Fig. 19 (right) and a uniform E63 airfoil shape transitioning to an E856 airfoil towards the hub. Both chord distribution and leading edge curve are generated as described by Ning [21], and shown in Fig. 19 (left), where $c$ is the chord length, $x$ is the distance from the centerline as shown in Fig. 18, and $z$ is the distance from the plane of rotation. All simulations are run at 4860 RPM and no freestream velocity, matching the experimental configuration resulting in a diameter-based Reynolds number $\operatorname{Re}_{D}$ of $6.5 \times 10^{5}$ and chord-based $\operatorname{Reynolds}$ of $\operatorname{Re}_{c}$ of

Table 1 Operation configuration of test cases.

\begin{tabular}{rccc}
\hline & DJI Phantom II & APC 10x7 & E779a \\
\hline Diameter & 9.4 in $(240 \mathrm{~mm})$ & $10 \mathrm{in}(254 \mathrm{~mm})$ & $8.9 \mathrm{in}(227 \mathrm{~mm})$ \\
Number of blades & 2 & 2 & 3 \\
Solidity & 0.12 & 0.10 & 0.40 \\
RPM & 4860 & $\sim 9200$ & 1500 \\
Tip speed & $0.18 \mathrm{Mach}$ & $0.36 \mathrm{Mach}$ & $0.05 \mathrm{Mach}$ \\
Freestream & $0 \mathrm{~m} / \mathrm{s}$ & $0-29 \mathrm{~m} / \mathrm{s}$ & $2.5-3.5 \mathrm{~m} / \mathrm{s}$ \\
Advance ratio & 0 & $0-0.75$ & $0.45-0.65$ \\
Chord-based Reynolds number & $6.2 \times 10^{4}$ & $1.2 \times 10^{5}$ & $8.9 \times 10^{5}$ \\
Diameter-based Reynolds number & $6.5 \times 10^{5}$ & $1.5 \times 10^{6}$ & $2.7 \times 10^{6}$ \\
\hline
\end{tabular}

\footnotetext{
${ }^{\dagger}$ https://github.com/byuflowlab/CCBlade.jl
} 
$6.2 \times 10^{4}$. Reynolds numbers are calculated at the $70 \%$ blade span as

$$
\operatorname{Re}_{D}=\frac{V_{70 \%} D}{v} \text { and } \operatorname{Re}_{c}=\frac{V_{70 \%} \bar{c}}{v},
$$

where $\bar{c}$ is the mean chord, and $V_{70 \%}$ is the effective speed resulting from both freestream and local rotational velocity at $70 \%$ the blade span.

The forward-flight configuration uses the APC thin-electric 10x7 propeller operating at a moderate Reynolds number. This propeller is a hobby-grade model commonly used in small unmanned aircraft, it is readily available, and its 10-inch diameter makes it a good fit for mid-size wind tunnel testing. Moreover, its performance has been measured and indexed in the UIUC Propeller Database [89], and verified by McCrink and Gregory [90] both experimentally and through blade-element momentum theory. The blade is generated assuming a Clark Y airfoil section from 0 to 5\% span, and a NACA 4412 for the remainder of the span, while using the chord and twist distributions digitized by McCrink and Gregory shown in Fig. 19. The leading edge curve was digitized by cutting slices of the actual blade. Note that forward flight in distributed-propulsion aircraft implies a fully axial inflow instead of the edgewise flow typically referred to in rotorcraft terminology. The performance of the individual propeller is validated against wind-tunnel measurements [90] across a sweep of advance ratio $J$ at a constant $\operatorname{Re}_{D}$ of $1.5 \times 10^{6}$ and $\operatorname{Re}_{c}$ of $1.2 \times 10^{5}$, where $J$ is calculated as $J=V_{\infty} /(n D)$, with $n=\mathrm{RPM} / 60$. Both $V_{\infty}$ and $n$ are varied accordingly to achieve the desired advance ratio and Reynolds number.
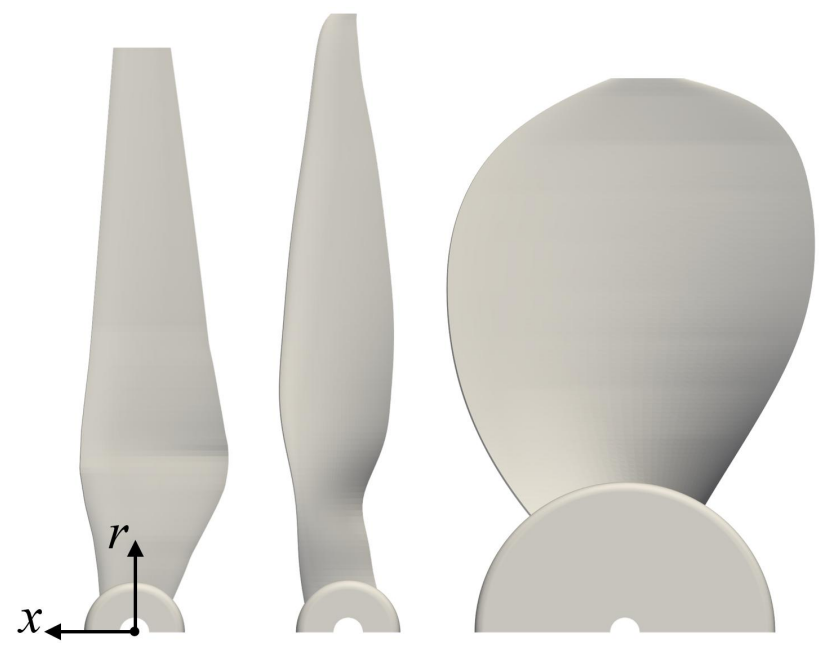

Fig. 18 Blade geometry of rotors simulated in this study. Left to right: DJI Phantom II, APC 10x7, E779a.
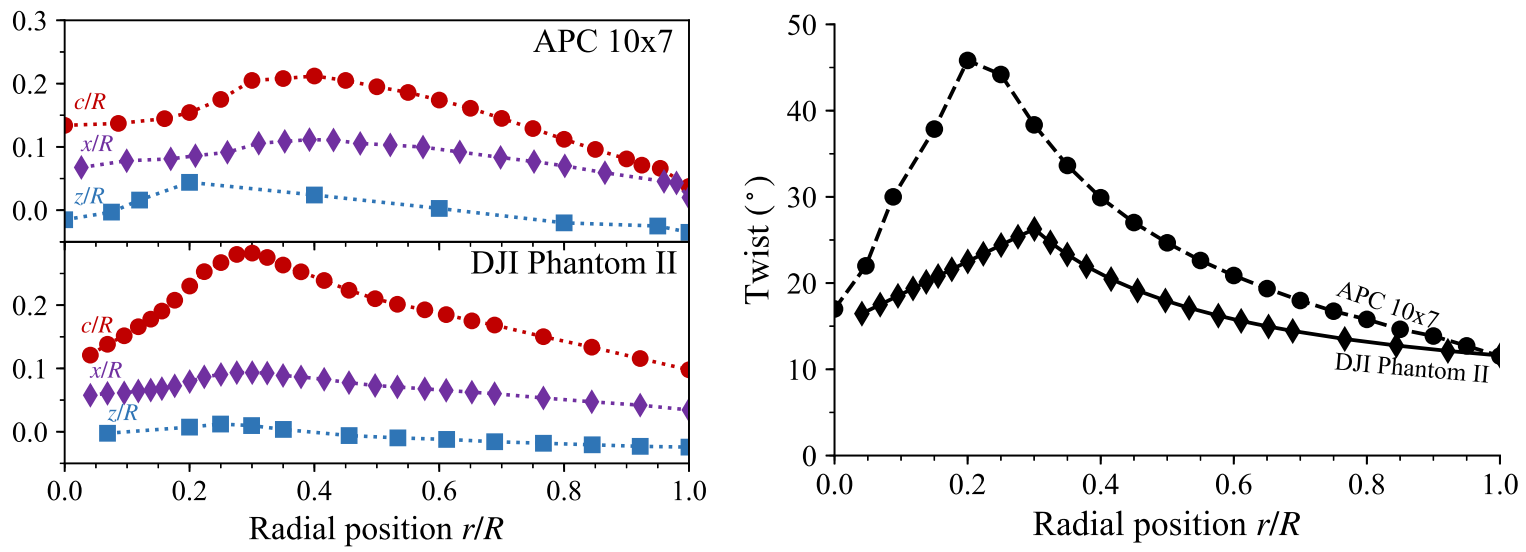

Fig. 19 Chord distribution and leading edge curve (left) of APC 10x7 and DJI Phantom II rotors, and corresponding twist distributions (right). 
Finally, high-Reynolds operation and wake dynamics are validated by comparison to experimental measurements on the marine propeller INSEAN E799a reported by Felli et al. [84] Underwater experimentation enables the visualization of tip vortices through cavitation bubbles, while reaching a high-Reynolds-number operation at a low tip Mach number. This subscale propeller is $227 \mathrm{~mm}$ in diameter, and its geometry was made available by INSEAN upon request. Thrust predictions are compared to reported measurements at $J=0.65$ and $\operatorname{Re}_{D}=2.7 \times 10^{6}\left(\right.$ or $\left.\operatorname{Re}_{c}=8.9 \times 10^{5}\right)$, while wake dynamics are qualitatively compared to visualizations at $J=0.45,0.55$, and 0.65 .

The thrust coefficient $C_{T}$, torque coefficient $C_{Q}$, and propulsive efficiency $\eta$ hereby reported are defined as

$$
C_{T}=\frac{T}{\rho n^{2} D^{4}}, \quad C_{Q}=\frac{Q}{\rho n^{2} D^{5}}, \text { and } \quad \eta=\frac{T V_{\infty}}{2 \pi n Q} .
$$

Unless otherwise indicated, all simulations are run at a time step equivalent to $5^{\circ}$ of rotation $\left(N_{\text {steps }}=72\right)$, particles are shed every $2.5^{\circ}$ of rotation $\left(N_{\text {sheds }}=144\right)$ with a tip core overlap $\lambda$ of 2.125 , and 50 blade elements per blade. As indicated by the convergence study in Section III.D, the numerical error of the simulation at a high advance ratio is estimated to be less than $1 \%$ for $C_{T}$.

In the following sections, the propeller model is first validated in a single-rotor configuration at low, moderate, and high Reynolds number in both hover (Section IV.B) and forward flight (Section IV.C). Finally, validation of predicted rotor-on-rotor interactions in two side-by-side rotors in hover is shown in Section IV.D.

\section{IV.B Single-rotor Results: Hover Case}

The hover configuration uses the DJI Phantom II rotor operating at a low Reynolds number $\left(\operatorname{Re}_{c}=6.2 \times 10^{4}\right)$ and constant 4860 RPM. The thrust coefficient $C_{T}$ of the rotor throughtout the simulation is shown in Fig. 10 along with the experimental mean thrust and fluctuation. The wake starts to develop and convect downstream during the first two iterations, and the near field becomes fully developed after only three iterations as indicated by the convergence of thrust. The periodic spikes observed at six, ten, and fourteen revolutions correspond to every instance when the turbulent breakdown region has been trimmed. The wake after eighteen revolutions is shown in Fig. 20 (left), where features of the topology can be clearly identified: a well-defined structure in the near field $(z<0.5 D)$, an onset of leapfrogging at $z \approx 0.5 D$, and a transition into turbulent breakdown at $z \approx 1.0 D$. The simulation converges to a mean $C_{T}$ of 0.1013 and a steady fluctuation (standard deviation) of 0.001, meanwhile Zhou et al. [20] and Ning [21] report an experimental mean $C_{T}$ of 0.1007 and a fluctuation of 0.008 . This results in an error of only $0.5 \%$ between the simulation and experimental mean $C_{T}$, meanwhile the fluctuation is largely underpredicted. We conjecture that the fluctuations
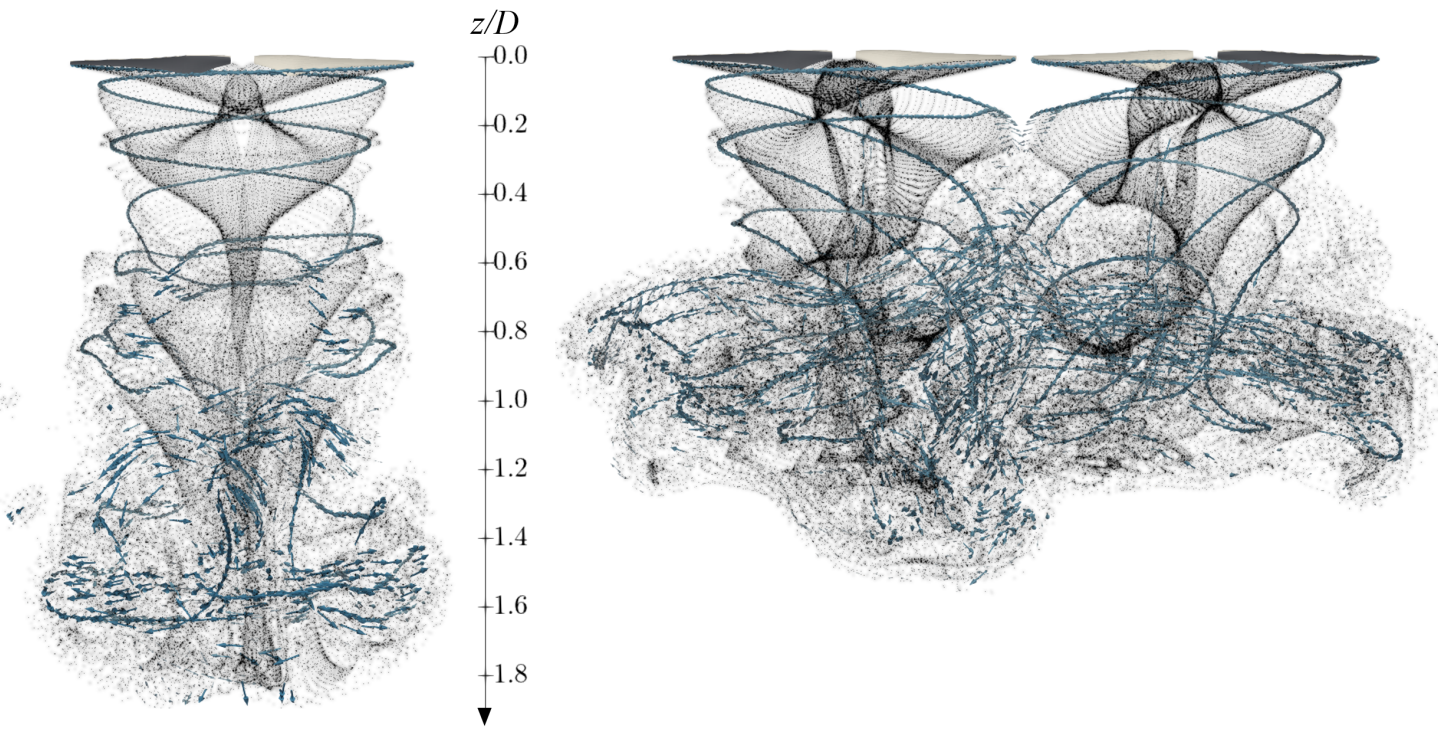

Fig. 20 Wake of single-rotor and multirotor hover simulations. Arrows scaled by the vortex strength of every particle. 

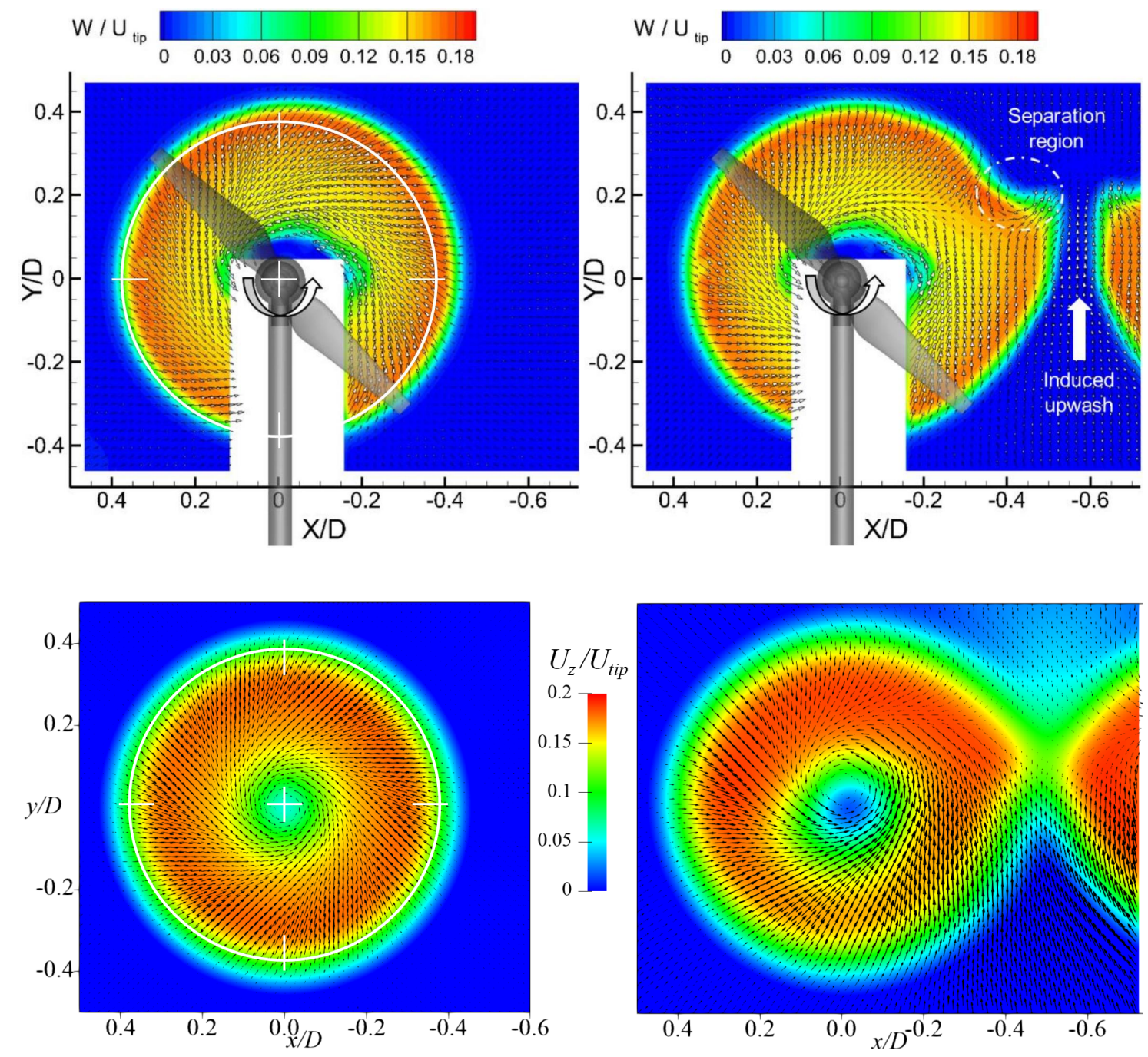

Fig. 21 Velocity field at plane $z=0.1 D$ of single rotor (left) and multirotor (right, $s=0.05 D$ ) in hover configuration. Top row shows the experimental ensemble average (retrieved from Zhou et al. [20]), bottom row shows the simulation ensemble average. Colormap corresponds to axial velocity, while arrows indicate swirl velocity.

observed in the experimental measurements of the individual rotor are enhanced by interactions with the test stand not captured in our simulations (e.g., mounting pole, hub, and mechanical vibrations).

Fig. 21 compares the ensemble-average velocity of the single rotor (left figures) observed experimentally and predicted at a plane located a distance $0.1 D$ downstream. The simulation shows a streamtube slightly more expanded than what is observed experimentally. The white circle centered at the hub shows that the experimental streamtube is not centered about the axis of rotation, which supports our belief that the experiment encounters significant interactions with the mounting pole. Fig. 22 shows the in-plane vorticity of both the experimental PIV and the simulated single rotor (left figures). The vorticity distribution is obtained as the average field at a phase-locked angle of $120^{\circ}$ over four revolutions, where the phase is defined as the angle between the vertical $y z$-plane and the position of the rotor blade. The VPM simulation shows a vortex core that is more diffused than what is observed experimentally. It must be noted that this vortex core matches the size of the smoothing radius $\sigma$ associated to the prescribed spatial discretization, and we suspect that a more accurate prediction could be achieved with a higher spatial resolution. In spite of that, the VPM simulation correctly predicts an onset of tip-vortex leapfrogging at a downstream distance $z \approx 0.5 D$. Thus, these results confirm that the propeller model captures all the physics of interest at low Reynolds number in a hovering single-rotor configuration. 

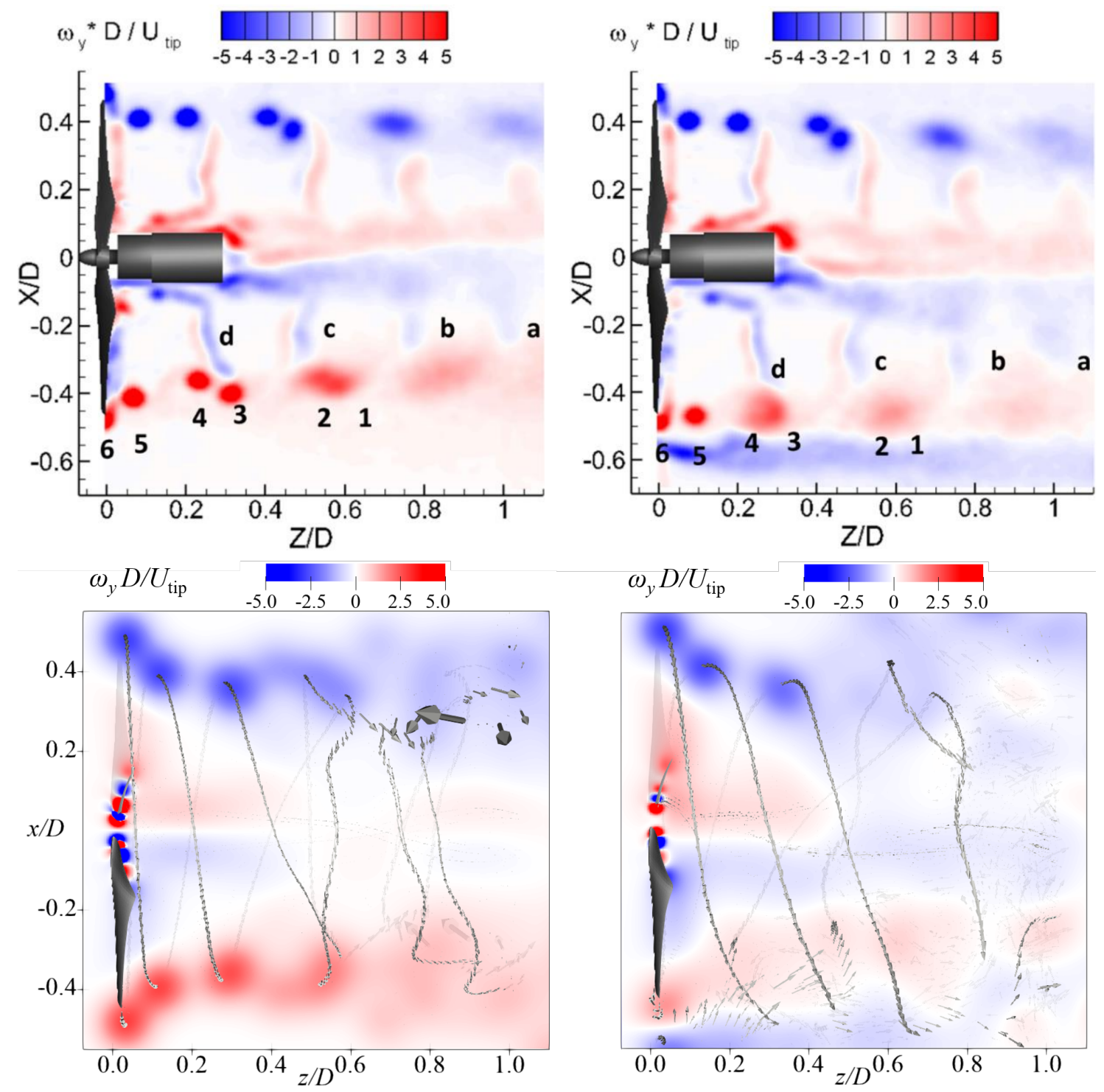

Fig. 22 Phase-locked average vorticity distribution of single rotor (left) and multirotor (right, $s=0.05 D$ ) in hover configuration as measured experimentally (top) and simulated (bottom). Arrows scaled by the vortex strength of every particle. Experimental images retrieved from Zhou et al. [20] 


\section{IV.C Single-rotor Results: Forward-flight Case}

The forward-flight configuration uses the APC 10x7 propeller operating at a moderate Reynolds number (constant $\operatorname{Re}_{c}$ of $1.2 \times 10^{5}$ ) across a sweep of advance ratio $J$. Fig. 23 compares the predicted thrust, torque, and propulsive efficiency to the experimental values reported by McCrink and Gregory [90]. It is observed that both $C_{T}$ and $C_{Q}$ match the experimental values at low and moderate advance ratios, but they drop earlier than what is seen experimentally at high advance ratios. This is also observed in the blade-element momentum predictions by McCrink and Gregory, which hints that this may be an issue with the geometry description (i.e., inaccurate airfoil shape, twist distribution, or aeroelastic twist caused by blade deflection at high advance ratios) rather than the modeling method. The discrepancies in $C_{T}$ and $C_{Q}$ cancel out as the propulsive efficiency $\eta$ is calculated, resulting in a good agreement across all advance ratios up to $J \approx 0.725$. Thus, it is confirmed that the VPM propeller model is valid at a moderate Reynolds number across low and moderately-high advance ratios in forward-flight single-rotor configuration.

In order to validate the predicted wake dynamics in forward flight, the E779a marine propeller was simulated at advance ratios $J=0.55$ and $J=0.65$. This underwater test case also provides validation of performance predictions at a high Reynolds number $\left(\operatorname{Re}_{c}=8.9 \times 10^{5}\right)$. The simulation at $J=0.65$ predicts a $C_{T}$ and $C_{Q}$ of 0.185 and 0.032 , respectively, meanwhile Felli et al. [84] report an experimental $C_{T}$ and $C_{Q}$ of 0.187 and 0.031 . This results in a discrepancy of $1.4 \%$ and $1.1 \%$ between experimental and predicted $C_{T}$ and $C_{Q}$, respectively. Fig. 24 compares the experimental wake visualized through cavitation bubbles and the simulation. In the case $J=0.55$, we see that the simulation predicts similar dynamics of leapfrogging and transition into turbulent breakdown as is observed experimentally, with these dynamics moving further downstream as $J$ is increased to 0.65 . Thus, it is confirmed that the
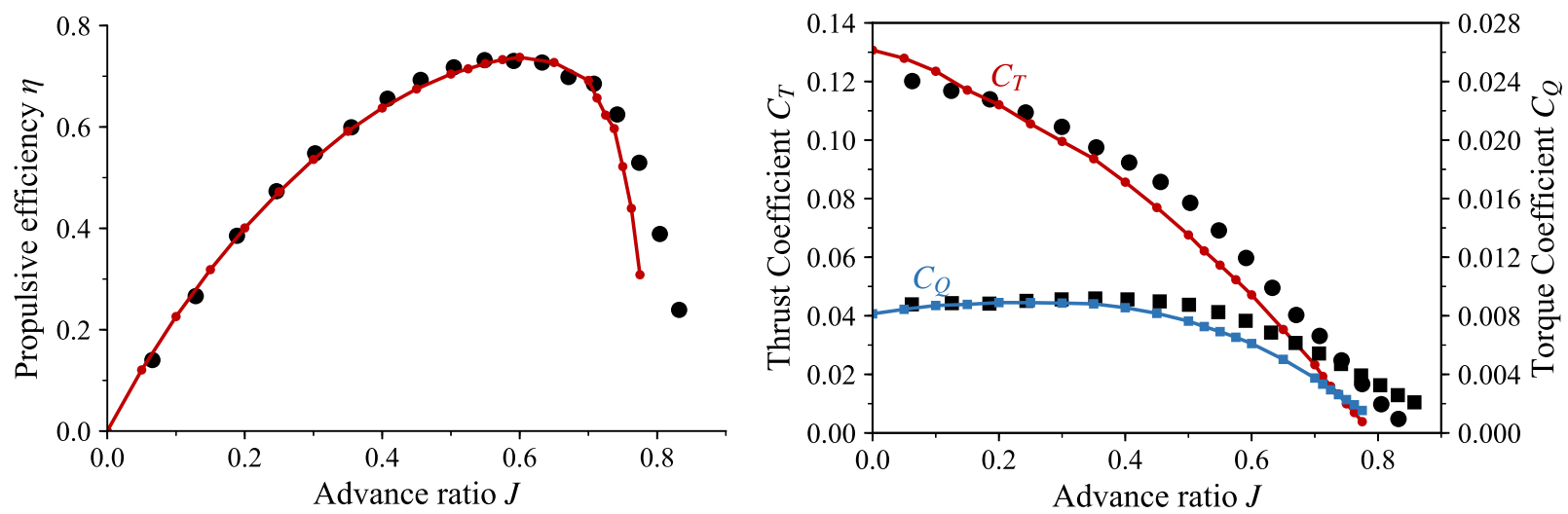

Fig. 23 Comparison between simulation (solid lines) and experimental (black markers) thrust, torque, and propulsive efficiency in forward-flight single-rotor configuration.

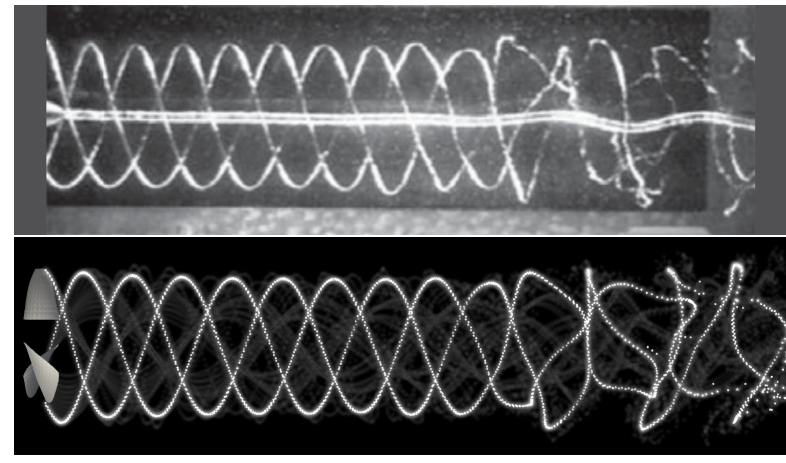

(a) $J=0.55$

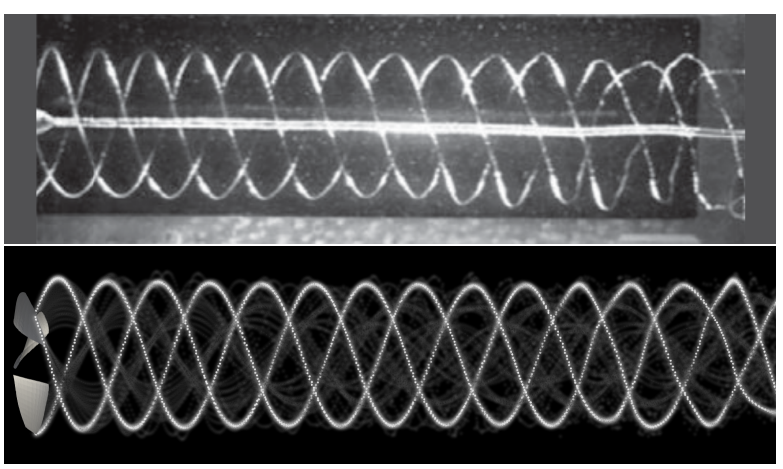

(b) $J=0.65$

Fig. 24 Comparison between experimental (top) and simulated (bottom) wake structure of the marine propeller E779a (top images reproduced by permission of Cambridge University Press from Felli et al. [84]). 
VPM propeller model provide accurate performance predictions at a high Reynolds number, while qualitative inspection indicates similar wake dynamics between simulation and experiment in forward flight.

\section{IV.D Multirotor Results}

The hovering single-rotor case of Section IV.B is now extended to multirotor configuration by adding an identical rotor in counter-rotation at a tip-to-tip distance $s$ of $0.05 \mathrm{D}$. Counter-rotation means that one propeller rotates clockwise, meanwhile the other rotates counter-clockwise. The simulation shows that the multirotor wake mixing onsets a topological instability that moves the turbulent-breakdown region closer to the plane of rotation, as shown in Fig. 20 (right). Fig. 22 compares the in-plane vorticity field between single-rotor and multirotor configuration. Both simulation and experiment show that tip vortices lose their structure as wakes mix in-between the rotors $(x=-0.5 D$ region, compare left and right figures).

Next, the separation distance $s$ was varied to determine its effects on thrust. Every time that blades pass through the region of wake mixing, the loading of the blade drops, decreasing the mean thrust and increasing the fluctuation, which is accentuated as the tip-to-tip distance becomes small. Fig. 25 shows the mean thrust (left) and thrust fluctuation (right) compared to what Zhou et al. [20] observed experimentally, normalized by their corresponding values in single-rotor configuration. Here it is seen that the simulation captures both the thrust drop and the increased fluctuation, showing satisfactory agreement with the experimental measurements. Thus, it is confirmed that the VPM multirotor model is able to capture aerodynamic rotor-on-rotor interactions. Furthermore, it is inferred that the unsteady loading introduced by these interactions are responsible for the increased acoustic noise reported by Zhou et al. [20], making the VPM a good candidate for a future application in noise prediction.

Zhou et al. observed an accentuated upwash and a recirculation region in the near field between the rotors (shown in Fig. 21, top right), and conjectured that the flow separates along the blades every time they pass through this region, leading to the observed drop in blade loading. The simulation (Fig. 21, bottom right) shows the same accentuated upwash observed experimentally, though the recirculation region is absent. Since the drop in thrust shown in Fig. 25 agrees well with the experiment in spite of not capturing the recirculation region, we conclude that the effects of the recirculation pocket are negligible and that the induced upwash is the main contributor to the drop in blade loading.

\section{Conclusion}

In this study we have shown the capability of the viscous vortex particle method to capture multirotor aerodynamic interactions. A VPM-based multirotor model was introduced along with recommendations for computational efficiency and an implementation achieving numerically stable simulations. A detailed convergence study was performed on four
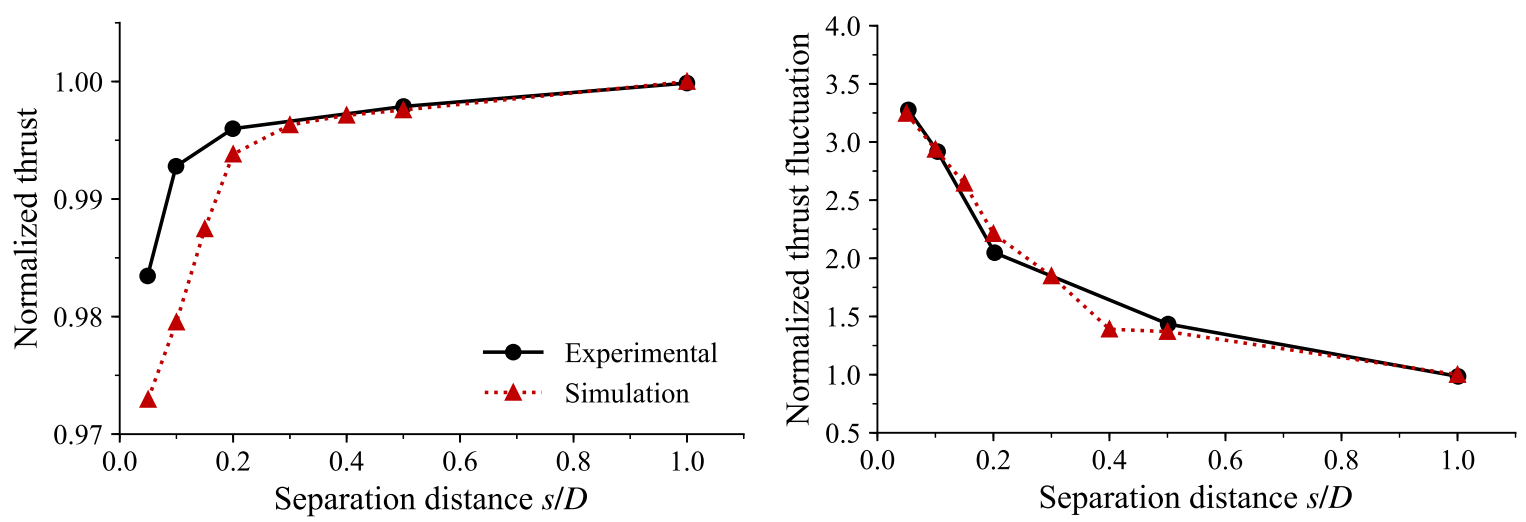

Fig. 25 Effects of rotor-on-rotor interactions on thrust as separation between rotors is decreased, normalized by their respective values in single-rotor configuration.

\footnotetext{
${ }^{\ddagger}$ Fluctuations are underpredicted as the experimental measurements include interactions inherent to the experimental setup (mounting pole and hub) not captured in the simulation, as mentioned in Section IV.B. However, simulation and experiment show good agreement after normalizing both by their respective single-rotor values.
} 
parameters characterizing temporal and spatial resolutions, showing a numerical error smaller than $1 \%$ at a high advance ratio. Single-rotor validation was presented at low, moderate, and high Reynolds numbers, showing an error of only $0.5 \%$ between experimental and predicted thrust coefficient in hover, and good agreement on predicted propulsive efficiency across low and moderately-high advance ratios in forward flight configuration. Wake dynamics in multirotor hovering configuration showed a good qualitative agreement between simulation and particle-image velocimetry measurements. The simulation indicates that the accentuated induced upwash in between the rotors is the main contributor to the drop in blade loading during rotor-on-rotor interaction. The multirotor VPM model showed good quantitative agreement with experimental thrust measurements, capturing the thrust drop observed experimentally as the tip-to-tip distance between rotors became small, along with the associated unsteady loading.

The validation of the VPM multirotor model hereby presented lays the groundwork for the future development of an aeroacoustic model predicting the noise associated to unsteady loading in multirotor configurations, with the potential of integrating these analyses in the conceptual design stage of multirotor aircraft.

\section{Acknowledgments}

This work is funded by the High Impact Doctoral Research fellowship granted by Brigham Young University. FLOWVPM uses a modified version of the open-source code ExaFMM originally developed by Lorena Barba and Rio Yokota. FLOWVPM integrates the open-source software Paraview as its visualization engine.

\section{References}

[1] Silva, C., Johnson, W. R., Antcliff, K. R., and Patterson, M. D., "VTOL Urban Air Mobility Concept Vehicles for Technology Development," 2018 Aviation Technology, Integration, and Operations Conference, 2018, pp. 1-16. doi:10.2514/6.2018-3847, URL https: //doi .org/10.2514/6.2018-3847.

[2] Moore, M., "NASA Puffin Electric Tailsitter VTOL Concept," 10th AIAA Aviation Technology, Integration, and Operations (ATIO) Conference, American Institute of Aeronautics and Astronautics, Reston, Virigina, 2010, pp. 1-12. doi:10.2514/6.20109345, URL http://arc.aiaa.org/doi/10.2514/6.2010-9345.

[3] Kasliwal, A., Furbush, N. J., Gawron, J. H., McBride, J. R., Wallington, T. J., De Kleine, R. D., Kim, H. C., and Keoleian, G. A., "Role of flying cars in sustainable mobility," Nature Communications, Vol. 10, No. 1, 2019 , p. 1555. doi:10.1038/s41467-019-09426-0, URL http://www .nature.com/articles/s41467-019-09426-0.

[4] Moore, K. R., and Ning, A., "Takeoff and Performance Trade-Offs of Retrofit Distributed Electric Propulsion for Urban Transport," Journal of Aircraft, Vol. 56, No. 5, 2019, pp. 1880-1892. doi:10.2514/1.C035321, URL https: //arc . aiaa . org/doi/10.2514/1.c035321.

[5] "Flying Cars: Investment Implications of Autonomous Urban Air Mobility," Tech. rep., Morgan Stanley Research, 2018. URL https://www.morganstanley.com/ideas/autonomous-aircrafthttps://www.geekwire.com/2018/ morgan-stanley-report-says-market-self-flying-cars-hit-1-5-trillion-2040/.

[6] Brelje, B. J., and Martins, J. R., "Electric, hybrid, and turboelectric fixed-wing aircraft: A review of concepts, models, and design approaches," Progress in Aerospace Sciences, , No. June, 2018, pp. 1-19. doi:10.1016/j.paerosci.2018.06.004, URL https://doi .org/10.1016/j.paerosci.2018.06.004.

[7] Pascioni, K., and Rizzi, S. A., "Tonal Noise Prediction of a Distributed Propulsion Unmanned Aerial Vehicle," 2018 AIAA/CEAS Aeroacoustics Conference, American Institute of Aeronautics and Astronautics, Reston, Virginia, 2018 , pp. 1-18. doi:10.2514/6.2018-2951, URL https://arc.aiaa.org/doi/10.2514/6.2018-2951.

[8] Ingraham, D., Gray, J. S., and Lopes, L. V., “Gradient-Based Propeller Optimization with Acoustic Constraints,” AIAA Scitech 2019 Forum, American Institute of Aeronautics and Astronautics, Reston, Virginia, 2019, pp. 1-12. doi:10.2514/6.2019-1219, URL https: //arc . aiaa.org/doi/10.2514/6.2019-1219.

[9] Persson, L., and Lawrence, B., "Design and Control of an Experimental Tiltwing Aircraft," Tech. rep., NASA, 2017.

[10] Droandi, G., Syal, M., and Bower, G., "Tiltwing Multi-Rotor Aerodynamic Modeling in Hover, Transition and Cruise Flight Conditions," AHS International 74th Annual Forum \& Technology Display, 2018, p. 2018. 
[11] Droandi, G., Gibertini, G., Grassi, D., Campanardi, G., and Liprino, C., "Proprotor-wing aerodynamic interaction in the first stages of conversion from helicopter to aeroplane mode," Aerospace Science and Technology, Vol. 58, 2016, pp. 116-133. doi: 10.1016/j.ast.2016.08.013, URL http://dx.doi.org/10.1016/j . ast.2016.08.013https://linkinghub.elsevier . com/retrieve/pii/S127096381630462X.

[12] Borer, N. K., Derlaga, J. M., Deere, K. A., Carter, M. B., Viken, S., Patterson, M. D., Litherland, B., and Stoll, A., “Comparison of Aero-Propulsive Performance Predictions for Distributed Propulsion Configurations," 55th AIAA Aerospace Sciences Meeting, 2017, pp. 1-16. doi:10.2514/6.2017-0209, URL http://arc .aiaa.org/doi/10.2514/6.2017-0209.

[13] Theodore, C. R., "A Summary of the NASA Design Environment for Novel Vertical Lift Vehicles (DELIVER) Project," AHS Paper 20180001325, 2018. URL https://rotorcraft.arc.nasa.gov/Publications/files/ TheodoreC\{_\}2018\{_\}TechMx.pdf.

[14] Ventura Diaz, P., and Yoon, S., "High-Fidelity Computational Aerodynamics of Multi-Rotor Unmanned Aerial Vehicles," 2018 AIAA Aerospace Sciences Meeting, 2018, pp. 1-22. doi:10.2514/6.2018-1266, URL https://arc .aiaa.org/doi/10. $2514 / 6.2018-1266$.

[15] Vegh, J. M., Botero, E., Clarke, M., Trent, J., and Alonso, J., "Current Capabilities and Challenges of NDARC and SUAVE for eVTOL Aircraft Design and Analysis," AIAA Propulsion and Energy 2019 Forum, American Institute of Aeronautics and Astronautics, Reston, Virginia, 2019, pp. 1-15. doi:10.2514/6.2019-4505, URL https://arc .aiaa.org/doi/10.2514/6. $2019-4505$.

[16] Shukla, D., Hiremath, N., and Komerath, N. M., "Low Reynolds Number Aerodynamics Study on Coaxial and Quad-Rotor," 2018 Applied Aerodynamics Conference, 2018. doi:10.2514/6.2018-4118, URL https://arc . aiaa.org/doi/10.2514/6.20184118.

[17] Shukla, D., and Komerath, N., “Multirotor Drone Aerodynamic Interaction Investigation,” Drones, Vol. 2, No. 4, 2018 , p. 43. doi:10.3390/drones2040043, URL http: //www . mdpi . com/2504-446X/2/4/43.

[18] Shukla, D., and Komerath, N., "Low Reynolds number multirotor aerodynamic wake interactions," Experiments in Fluids, Vol. 60, No. 4, 2019, p. 77. doi:10.1007/s00348-019-2724-3, URL http://link. springer . com/10.1007/s00348-019-2724-3.

[19] Veismann, M., and Gharib, M., "High Fidelity Aerodynamic Force Estimation for Multirotor Crafts in Free Flight," AIAA Scitech 2020 Forum, American Institute of Aeronautics and Astronautics, Reston, Virginia, 2020, pp. 1-17. doi:10.2514/6.2020-0303, URL https://arc . aiaa.org/doi/10.2514/6.2020-0303.

[20] Zhou, W., Ning, Z., Li, H., and Hu, H., “An Experimental Investigation on Rotor-to-Rotor Interactions of Small UAV Propellers," 35th AIAA Applied Aerodynamics Conference, , No. June, 2017, pp. 1-16. doi:10.2514/6.2017-3744, URL https://arc.aiaa.org/doi/10.2514/6.2017-3744.

[21] Ning, Z., "Experimental investigations on the aerodynamic and aeroacoustic characteristics of small UAS propellers," Ph.D. thesis, Iowa State University, Digital Repository, Ames, 2018. doi:10.31274/etd-180810-6057, URL http://arc . aiaa . org/ doi/10.2514/6.2016-1785https://lib.dr.iastate.edu/etd/16427/.

[22] Stokkermans, T. C. A., Usai, D., Sinnige, T., and Veldhuis, L. L. M., "Aerodynamic Interaction Effects between Propellers in Typical eVTOL Vehicle Configurations," Journal of Aircraft (in review), 2020.

[23] LEE, J., YEE, K., and OH, S., “Aerodynamic Characteristic Analysis of Multi-Rotors Using a Modified Free-Wake Method," TRANSACTIONS OF THE JAPAN SOCIETY FOR AERONAUTICAL AND SPACE SCIENCES, Vol. 52, No. 177, 2009, pp. 168-179. doi:10.2322/tjsass.52.168, URL http://joi . jlc.jst.go . jp/JST . JSTAGE/tjsass/52 . 168? from=CrossRef.

[24] Yoon, S., Lee, H. C., and Pulliam, T. H., "Computational Analysis of Multi-Rotor Flows,” 54th AIAA Aerospace Sciences Meeting, , No. January, 2016, pp. 1-11. doi:10.2514/6.2016-0812, URL http://arc . aiaa.org/doi/10.2514/6.2016-0812.

[25] Ventura, P., Yoon, S., and Theodore, C. R., "High-Fidelity Computational Aerodynamics of the Elytron 4S UAV," American Helicopter Society International - Future Vertical Lift Aircraft Design Conference, San Francisco CA, 2018.

[26] Abras, J., Hariharan, N. S., and Narducci, R. P., "Wake Breakdown of High-fidelity Simulations of a Rotor in Hover," AIAA Scitech 2019 Forum, American Institute of Aeronautics and Astronautics, Reston, Virginia, 2019, pp. 1-20. doi:10.2514/6.2019-0593, URL https://arc . aiaa.org/doi/10.2514/6.2019-0593.

[27] Wagner, L. N., “Concept Evaluation : NASA Reference Model 2 - Side by Side,” Tech. Rep. February, NASA, 2019. 
[28] Mankbadi, R. R., Afari, S., and Golubev, V. V., "Towards High-fidelity Analysis of Noise Radiation and Control of Propellerdriven UAV," 25th AIAA/CEAS Aeroacoustics Conference, American Institute of Aeronautics and Astronautics, Reston, Virginia, 2019, pp. 1-14. doi:10.2514/6.2019-2632, URL https://arc . aiaa.org/doi/10.2514/6.2019-2632.

[29] Ventura Diaz, P., Johnson, W., Ahmad, J., and Yoon, S., "The Side-by-Side Urban Air Taxi Concept," AIAA Aviation 2019 Forum, American Institute of Aeronautics and Astronautics, Reston, Virginia, 2019. doi:10.2514/6.2019-2828, URL https://arc.aiaa.org/doi/10.2514/6.2019-2828.

[30] Alvarez, E. J., and Ning, A., "Development of a Vortex Particle Code for the Modeling of Wake Interaction in Distributed Propulsion," 2018 Applied Aerodynamics Conference, American Institute of Aeronautics and Astronautics, 2018 , pp. 1-22. doi:10.2514/6.2018-3646, URL https://arc .aiaa.org/doi/10.2514/6.2018-3646.

[31] Winckelmans, G., and Leonard, A., "Contributions to Vortex Particle Methods for the Computation of Three-Dimensional Incompressible Unsteady Flows,” Journal of Computational Physics, Vol. 109, No. 2, 1993, pp. 247-273. doi:10.1006/jcph. 1993.1216, URL http://linkinghub.elsevier.com/retrieve/pii/S0021999183712167.

[32] Barba, L. A., Leonard, A., and Allen, C. B., “Advances in viscous vortex methods - Meshless spatial adaption based on radial basis function interpolation," International Journal for Numerical Methods in Fluids, Vol. 47, No. 5, 2005 , pp. 387-421. doi:10.1002/fld.811.

[33] Willis, D. J., “An Unsteady, Accelerated, High Order Panel Method with Vortex Particle Wakes,” Ph.D. thesis, Massachusetts Institute of Technology, 2006. URL http://hdl . handle.net/1721.1/35592.

[34] Willis, D., Peraire, J., and White, J., “A Combined pFFT - Multipole Tree Code, Unsteady Panel Method with Vortex Particle Wakes," 43rd AIAA Aerospace Sciences Meeting and Exhibit, American Institute of Aeronautics and Astronautics, Reston, Virigina, 2005. doi:10.2514/6.2005-854, URL http://arc . aiaa.org/doi/10.2514/6.2005-854.

[35] Calabretta, J. S., and Mcdonald, R. A., "A Three Dimensional Vortex Particle-Panel Method for Modeling Propulsion-Airframe Interaction," 48th AIAA Aerospace Sciences Meeting Including the New Horizons Forum and Aerospace Exposition, , No. January, 2010, p. 2010. doi:10.15368/theses.2010.104.

[36] Berdowski, T. J., "3D Lagrangian VPM-FMM for Modelling the Near-Wake of a HAWT," Thesis, Technical University of Denmark and Delft University of Technology, 2015.

[37] Teixeira, P., and Cesnik, C. E., "Propeller Effects on the Dynamic Response of HALE Aircraft," 2018 AIAA/ASCE/AHS/ASC Structures, Structural Dynamics, and Materials Conference, American Institute of Aeronautics and Astronautics, Reston, Virginia, 2018, pp. 1-15. doi:10.2514/6.2018-1202, URL https://arc . aiaa.org/doi/10.2514/6.2018-1202.

[38] Wang, H., Zhou, Z., Xu, X., and Zhu, X., "Influence analysis of propeller location parameters on wings using a panel/viscous vortex particle hybrid method," The Aeronautical Journal, Vol. 122, No. 1247, 2018, pp. 21-41. doi:10.1017/aer.2017.109, URL https: //www . cambridge.org/core/product/identifier/S0001924017001099/type/journal\{_\}article.

[39] Lee, H., and Lee, D.-J., "Effects of platform motions on aerodynamic performance and unsteady wake evolution of a floating offshore wind turbine," Renewable Energy, Vol. 143, 2019, pp. 9-23. doi:10.1016/j.renene.2019.04.134, URL https://linkinghub.elsevier.com/retrieve/pii/S0960148119306184.

[40] Sessarego, M., Feng, J., Ramos-García, N., and Horcas, S. G., "Design optimization of a curved wind turbine blade using neural networks and an aero-elastic vortex method under turbulent inflow," Renewable Energy, 2019. doi:10.1016/j.renene.2019. 07.046, URL https://doi.org/10.1016/j.renene.2019.07.046https://linkinghub.elsevier.com/retrieve/ $\mathrm{pii/S0960148119310663.}$

[41] Winckelmans, G., Cocle, R., Dufresne, L., and Capart, R., "Vortex methods and their application to trailing wake vortex simulations," Comptes Rendus Physique, Vol. 6, No. 4-5 SPEC. ISS., 2005, pp. 467-486. doi:10.1016/j.crhy.2005.05.001.

[42] Stock, M., Gharakhani, A., and Stone, C., "Modeling Rotor Wakes with a Hybrid OVERFLOW-Vortex Method on a GPU Cluster," 28th AIAA Applied Aerodynamics Conference, , No. July, 2010, pp. 1-12. doi:10.2514/6.2010-4553, URL http://arc.aiaa.org/doi/abs/10.2514/6.2010-4553.

[43] Chatelain, P., Duponcheel, M., Caprace, D.-G., Marichal, Y., and Winckelmans, G., "Vortex particle-mesh simulations of vertical axis wind turbine flows: from the airfoil performance to the very far wake," Wind Energy Science, Vol. 2, No. 1, 2017, pp. 317-328. doi:10.5194/wes-2-317-2017, URL http://stacks.iop.org/1742-6596/753/i=3/a=032007?key= crossref.fc2b2fe9996c69d4a419caec4e075875https://www.wind-energ-sci .net/2/317/2017/. 
[44] Yokota, R., Barba, L. A., Narumi, T., and Yasuoka, K., "Petascale turbulence simulation using a highly parallel fast multipole method on GPUs," Computer Physics Communications, Vol. 184, No. 3, 2013, pp. 445-455. doi:10.1016/j.cpc.2012.09.011, URL http://dx.doi.org/10.1016/j.cpc.2012.09.011.

[45] Yokota, R., and Barba, L. A., "FMM-based vortex method for simulation of isotropic turbulence on GPUs , compared with a spectral method," Computers and Fluids, Vol. 80, 2013, pp. 17-27. doi:10.1016/j.compfluid.2012.08.002, URL http://dx.doi.org/10.1016/j.compfluid.2012.08.002.

[46] Hu, Q., Gumerov, N. A., Yokota, R., Barba, L., and Duraiswami, R., "Scalable Fast Multipole Accelerated Vortex Methods," 2014 IEEE International Parallel \& Distributed Processing Symposium Workshops, IEEE, 2014, pp. 966-975. doi:10.1109/ IPDPSW.2014.110, URL http: //ieeexplore. ieee.org/document/6969486/.

[47] Alvarez, E. J., and Ning, A., "Modeling Multirotor Aerodynamic Interactions Through the Vortex Particle Method," AIAA Aviation 2019 Forum, American Institute of Aeronautics and Astronautics, Dallas, Texas, 2019. doi:10.2514/6.2019-2827, URL https: //arc . aiaa.org/doi/10.2514/6.2019-2827.

[48] Lee, D. J., and Na, S. U., "Numerical simulations of wake structure generated by rotating blades using a time marching, free vortex blob method," European Journal of Mechanics - B/Fluids, Vol. 18, No. 1, 1999, pp. 147-159. doi:10.1016/S09977546(99)80011-9, URL http://linkinghub.elsevier.com/retrieve/pii/S0997754699800119.

[49] Opoku, D. G., Triantos, D. G., Nitzsche, F., and Voutsinas, S. G., "Rotorcraft Aerodynamic and Aeroacoustic Modelling Using Vortex Particle Methods," ICAS 2002 Congress, , No. 1, 2002, pp. 299.1-299.11.

[50] Zhao, J., and He, C., "Enhancement of Viscous Vortex Particle Method for Fundamental Rotor Wake Dynamics Simulation," New Horizons, , No. January, 2012, pp. 1-11.

[51] Jo, Y., Lee, H., and Lee, D. J., "Prediction of rotor flow for unmanned aerial system using nonlinear vortex lattice method," 6th Asian-Australian Rotorcraft Forum and Heli Japan 2017, ARF 2017, 2017. doi:10.1016/j.jse.2010.09.008.

[52] Ho, J. C., and Yeo, H., “Assessing Calculated Blade Loads of the Tilt Rotor Aeroacoustic Model," Journal of Aircraft, Vol. 55, No. 3, 2018, pp. 1287-1298. doi:10.2514/1.C034624, URL https://arc.aiaa.org/doi/10.2514/1.CQ34624.

[53] Singh, P., and Friedmann, P. P., “A Computational Fluid Dynamics-Based Viscous Vortex Particle Method for Coaxial Rotor Interaction Calculations in Hover," Journal of the American Helicopter Society, , No. May, 2018. doi:10.4050/jahs.63.042002.

[54] Tan, J., Sun, Y., and Barakos, G., "Unsteady loads for coaxial rotors in forward flight computed using a vortex particle method," Aeronautical Journal, Vol. 122, No. 1251, 2018, pp. 693-714. doi:10.1017/aer.2018.8.

[55] Singh, P., and Friedmann, P. P., "Dynamic Stall modeling using Viscous Vortex Particle Method for Coaxial Rotors," Vertical Flight Society 75th Annual Forum \& Technology Display, 2019.

[56] He, C., and Zhao, J., "Modeling Rotor Wake Dynamics with Viscous Vortex Particle Method," AIAA Journal, Vol. 47, No. 4, 2009, pp. 902-915. doi:10.2514/1.36466, URL https://doi .org/10.2514/1.36466.

[57] Tan, J. F., and Wang, H. W., "Simulating unsteady aerodynamics of helicopter rotor with panel/viscous vortex particle method," Aerospace Science and Technology, Vol. 30, No. 1, 2013, pp. 255-268. doi:10.1016/j.ast.2013.08.010, URL http://dx.doi.org/10.1016/j.ast.2013.08.010.

[58] Shi, Y., Xu, G., and Wei, P., "Rotor wake and flow analysis using a coupled Eulerian-Lagrangian method," Engineering Applications of Computational Fluid Mechanics, Vol. 10, No. 1, 2016, pp. 384-402. doi:10.1080/19942060.2016. 1174887, URL http://dx.doi.org/10.1080/19942060.2016.1174887https://www . tandfonline.com/doi/full/ $10.1080 / 19942060.2016 .1174887$.

[59] Shi, Y., Xu, Y., Xu, G., and Wei, P., "A coupling VWM/CFD/CSD method for rotor airload prediction,” Chinese Journal of Aeronautics, Vol. 30, No. 1, 2017, pp. 204-215. doi:10.1016/j.cja.2016.12.014, URL http://linkinghub.elsevier .com/ retrieve/pii/S1000936116302333.

[60] Battey, L. S., “A Hybrid Navier Stokes/Vortex Particle Wake Methodology for Modeling Helicopter Rotors in Forward Flight and Maneuvers,” Thesis, Georgia Institute of Technology, 2018. URL http://hdl . handle . net/1853/59934.

[61] Tan, J. F., Sun, Y. M., and Barakos, G. N., "Vortex Approach for Downwash and Outwash of Tandem Rotors in Ground Effect," Journal of Aircraft, Vol. 55, No. 6, 2018, pp. 1-19. doi:10.2514/1.C034740, URL https://arc.aiaa.org/doi/10.2514/ 1. $\operatorname{CQ3} 4740$. 
[62] Tan, J. F., Zhou, T. Y., Sun, Y. M., and Barakos, G. N., "Numerical investigation of the aerodynamic interaction between a tiltrotor and a tandem rotor during shipboard operations," Aerospace Science and Technology, Vol. 87, 2019 , pp. 62-72. doi:10.1016/j.ast.2019.02.005, URL https://doi .org/10.1016/j . ast.2019.02.005.

[63] Tan, J. F., Sun, Y. M., Wang, H. W., and Lin, C. L., "New Approach for Aerodynamic and Aeroacoustic Analysis of Actively Controlled Flaps Rotor," Journal of Aircraft, Vol. 55, No. 6, 2018, pp. 2191-2202. doi:10.2514/1.C034225, URL https://arc.aiaa.org/doi/10.2514/1.C034225.

[64] Jo, Y., Jardin, T., Gojon, R., Jacob, M. C., and Moschetta, J.-M., "Prediction of Noise from Low Reynolds Number Rotors with Different Number of Blades using a Non-Linear Vortex Lattice Method," 25th AIAA/CEAS Aeroacoustics Conference, , No. May, 2019, pp. 1-13. doi:10.2514/6.2019-2615, URL https://arc . aiaa.org/doi/10. 2514/6.2019-2615.

[65] Zolbayar, B.-E., "Investigation of Noise From Electric, Low-Tip-Speed Aircraft Propellers," The Pennsylvania State University, , No. August, 2018.

[66] Winckelmans, G. S., "Topics in vortex methods for the computation of three and two dimensional incompressible unsteady flows,", 1989. URL http://core.kmi.open.ac.uk/download/pdf/11813390.pdf.

[67] Barnes, J., and Hut, P., “A hierarchical O(N $\log \mathrm{N})$ force-calculation algorithm,” Nature, Vol. 324, No. 6096, 1986 , pp. $446-449$. doi:10.1038/324446a0, URL http://www . nature.com/doifinder/10.1038/324446a0.

[68] Greengard, L., Rokhlin, V., Rokhlin, L. G., and V., "A fast algorithm for particle simulations," Journal of Computational Physics, Vol. 73, No. 2, 1987, pp. 325-348. doi:10.1016/0021-9991(87)90140-9, URL http://ac.els-cdn.com/0021999187901409/1-s2.0-0021999187901409-main.pdf?\{_\}tid=679b26b05ae4-11e5-8234-00000aacb361\{\&\}acdnat=1442237454\{_\}f6867439347a63629fe376b91185a193\{\%\}5Cnhttp: //wwW.sciencedirect.com/science/article/pii/0021999187901409.

[69] Greengard, L. F., “The Rapid Evaluation Of Potential Fields In Particle Systems,” Phd thesis, Yale University, 1987.

[70] Cheng, H., Greengard, L., and Rokhlin, V., "A Fast Adaptive Multipole Algorithm in Three Dimensions," Journal of Computational Physics, Vol. 155, No. 2, 1999, pp. 468-498. doi:10.1006/jcph. 1999.6355, URL http://www.sciencedirect.com/science/article/pii/S0021999199963556\{\%\}5Cnhttp:// linkinghub.elsevier.com/retrieve/pii/S0021999199963556.

[71] Yokota, R., and Barba, L. A., "Treecode and fast multipole method for N-body simulation with CUDA," GPU Computing Gems Emerald Edition, 2011, pp. 113-132. doi:10.1016/B978-0-12-384988-5.00009-7.

[72] Yokota, R., and Barba, L. A., "Comparing the treecode with FMM on GPUs for vortex particle simulations of a leapfrogging vortex ring," Computers and Fluids, Vol. 45, No. 1, 2011, pp. 155-161. doi:10.1016/j.compfluid.2010.11.029.

[73] Squire, W., and Trapp, G., "Using complex variables to estimate derivatives of real functions," SIAM Review, Vol. 40, No. 1, 1998, pp. 110-112. doi:10.1137/S003614459631241X.

[74] Yokota, R., and Barba, L. A., Treecode and fast multipole method for N-body simulation with CUDA, NVIDIA Corporation and Wen-mei W. Hwu, 2011. doi:10.1016/B978-0-12-384988-5.00009-7, URL http://dx . doi .org/10. 1016/B978-0-12384988-5.00009-7.

[75] Rossi, L. F., "Resurrecting Core Spreading Vortex Methods: A New Scheme that is Both Deterministic and Convergent," SIAM Journal on Scientific Computing, Vol. 17, No. 2, 1996, pp. 370-397. doi:10.1137/S1064827593254397, URL http://epubs.siam.org/doi/10.1137/S1064827593254397.

[76] Barba, L. a., "Vortex Method for computing high-Reynolds number Flows: Increased accuracy with a fully mesh-less formulation," California Institute of Technology, Vol. 2004, 2004.

[77] Torres, C. E., and Barba, L. A., "Fast radial basis function interpolation with Gaussians by localization and iteration," Journal of Computational Physics, Vol. 228, No. 14, 2009, pp. 4976-4999. doi:10.1016/j.jcp.2009.03.007, URL http: //dx.doi.org/10.1016/j.jcp.2009.03.007.

[78] Pedrizzetti, G., “Insight into singular vortex flows," Fluid Dynamics Research, Vol. 10, No. 2, 1992, pp. 101-115. doi: 10.1016/0169-5983(92)90011-K.

[79] Williamson, J. H., "Low-storage Runge-Kutta schemes," Journal of Computational Physics, Vol. 35, No. 1, 1980 , pp. 48-56. doi:10.1016/0021-9991(80)90033-9. 
[80] Du, Z., and Selig, M., “A 3-D stall-delay model for horizontal axis wind turbine performance prediction,” 1998 ASME Wind Energy Symposium, American Institute of Aeronautics and Astronautics, Reston, Virigina, 1998. doi:10.2514/6.1998-21, URL http://arc.aiaa.org/doi/pdf/10.2514/6.1998-21http://arc. aiaa.org/doi/10.2514/6.1998-21.

[81] Viterna, L. A., and Janetzke, D. C., "Theoretical and experimental power from large horizontal-axis wind turbines," Tech. rep., Washington Procurement Operations Office, Washington, DC (United States), sep 1982. doi:10.2172/6763041, URL http://www.osti.gov/servlets/purl/6763041/.

[82] Lee, H., and Lee, D. J., "Numerical investigation of the aerodynamics and wake structures of horizontal axis wind turbines by using nonlinear vortex lattice method," Renewable Energy, Vol. 132, 2019, pp. 1121-1133. doi:10.1016/j.renene.2018.08.087, URL https://doi .org/10.1016/j . renene.2018.08.087.

[83] Quaranta, H. U., Bolnot, H., and Leweke, T., "Long-wave instability of a helical vortex," Journal of Fluid Mechanics, Vol. 780, 2015, pp. 687-716. doi:10.1017/jfm.2015.479.

[84] Felli, M., Camussi, R., and Di Felice, F., "Mechanisms of evolution of the propeller wake in the transition and far fields," Journal of Fluid Mechanics, Vol. 682, 2011, pp. 5-53. doi:10.1017/jfm.2011.150.

[85] Gillis, T., Marichal, Y., Winckelmans, G., and Chatelain, P., “A 2D immersed interface Vortex Particle-Mesh method," Journal of Computational Physics, Vol. 394, 2019, pp. 700-718. doi:10.1016/j.jcp.2019.05.033, URL https://doi .org/10.1016/ j.jcp.2019.05.033https://linkinghub.elsevier.com/retrieve/pii/S0021999119303717.

[86] Giannopoulou, O., Colagrossi, A., Di Mascio, A., and Mascia, C., "Chorin's approaches revisited: Vortex Particle Method vs Finite Volume Method," Engineering Analysis with Boundary Elements, Vol. 106, No. May, 2019, pp. 371-388. doi: 10.1016/j.enganabound.2019.05.026, URL https://linkinghub. elsevier . com/retrieve/pii/S0955799719302164.

[87] Sullivan, I. S., Niemela, J. J., Hershberger, R. E., Bolster, D., and Donnelly, R. J., "Dynamics of thin vortex rings," Journal of Fluid Mechanics, Vol. 609, 2008, pp. 319-347. doi:10.1017/S0022112008002292, URL http://www . journals . cambridge . org/abstract\{_\}S0022112008002292.

[88] Cottet, G.-H., and Koumoutsakos, P. D., Vortex Methods, Cambridge University Press, Cambridge, 2000. doi:10.1017/ CBO9780511526442, URL http://ebooks . cambridge.org/ref/id/CB09780511526442.

[89] Brandt, J. B., Deters, R. W., Ananda, G. K., and Selig, M. S., "UIUC Propeller Database," http://mselig.ae.illinois.edu/props/propDB.html, ???? URL http://m-selig.ae.illinois.edu/props/propDB.html.

[90] McCrink, M. H., and Gregory, J. W., "Blade Element Momentum Modeling of Low-Reynolds Electric Propulsion Systems," Journal of Aircraft, Vol. 54, No. 1, 2017, pp. 163-176. doi:10.2514/1.C033622, URL http://arc.aiaa.org/doi/10. $2514 / 1 . \operatorname{co3} 3622$. 